NASA/TM-2003-212495

Robust and Simple Non-Reflecting Boundary

Conditions for the Euler EquationsA New Approach Based on the Space-Time CE/SE Method

S.C. Chang

Glenn Research Center, Cleveland, Ohio

A. Himansu, C.Y. Loh, and X.Y. Wang

Taitech, Inc., Beaver Creek, Ohio

S.T. Yu

Wayne State University, Detroit, Michigan 
Since its founding, NASA has been dedicated to the advancement of aeronautics and space science. The NASA Scientific and Technical Information (STI) Program Office plays a key part in helping NASA maintain this important role.

The NASA STI Program Office is operated by Langley Research Center, the Lead Center for NASA's scientific and technical information. The NASA STI Program Office provides access to the NASA STI Database, the largest collection of aeronautical and space science STI in the world. The Program Office is also NASA's institutional mechanism for disseminating the results of its research and development activities. These results are published by NASA in the NASA STI Report Series, which includes the following report types:

- $\quad$ TECHNICAL PUBLICATION. Reports of completed research or a major significant phase of research that present the results of NASA programs and include extensive data or theoretical analysis. Includes compilations of significant scientific and technical data and information deemed to be of continuing reference value. NASA's counterpart of peerreviewed formal professional papers but has less stringent limitations on manuscript length and extent of graphic presentations.

- TECHNICAL MEMORANDUM. Scientific and technical findings that are preliminary or of specialized interest, e.g., quick release reports, working papers, and bibliographies that contain minimal annotation. Does not contain extensive analysis.

- CONTRACTOR REPORT. Scientific and technical findings by NASA-sponsored contractors and grantees.
- CONFERENCE PUBLICATION. Collected papers from scientific and technical conferences, symposia, seminars, or other meetings sponsored or cosponsored by NASA.

- SPECIAL PUBLICATION. Scientific, technical, or historical information from NASA programs, projects, and missions, often concerned with subjects having substantial public interest.

- TECHNICAL TRANSLATION. Englishlanguage translations of foreign scientific and technical material pertinent to NASA's mission.

Specialized services that complement the STI Program Office's diverse offerings include creating custom thesauri, building customized databases, organizing and publishing research results ... even providing videos.

For more information about the NASA STI Program Office, see the following:

- Access the NASA STI Program Home Page at http://www.sti.nasa.gov

- E-mail your question via the Internet to help@sti.nasa.gov

- Fax your question to the NASA Access Help Desk at 301-621-0134

- Telephone the NASA Access Help Desk at 301-621-0390

- Write to:

NASA Access Help Desk

NASA Center for AeroSpace Information 7121 Standard Drive

Hanover, MD 21076 
NASA/TM-2003-212495

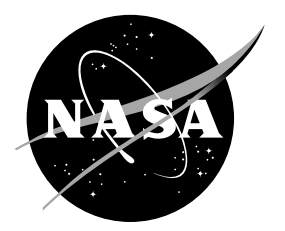

\section{Robust and Simple Non-Reflecting Boundary Conditions for the Euler Equations- A New Approach Based on the Space-Time CE/SE Method}

S.C. Chang

Glenn Research Center, Cleveland, Ohio

A. Himansu, C.Y. Loh, and X.Y. Wang

Taitech, Inc., Beaver Creek, Ohio

S.T. Yu

Wayne State University, Detroit, Michigan

National Aeronautics and

Space Administration

Glenn Research Center 
The Propulsion and Power Program at NASA Glenn Research Center sponsored this work.

Available from

NASA Center for Aerospace Information 7121 Standard Drive

Hanover, MD 21076
National Technical Information Service 5285 Port Royal Road Springfield, VA 22100

Available electronically at http:/ /gltrs.grc.nasa.gov 


\title{
Robust and Simple Non-Reflecting Boundary Conditions for the Euler Equations- A New Approach Based on the Space-Time CE/SE Method
}

\author{
S.C. Chang \\ National Aeronautics and Space Administration \\ Glenn Research Center \\ Cleveland, Ohio
}

\author{
A. Himansu, C.Y. Loh, and X.Y. Wang \\ Taitech, Inc. \\ Beaver Creek, Ohio
}

\author{
S.T. Yu \\ Wayne State University \\ Detroit, Michigan
}

\begin{abstract}
This paper reports on a significant advance in the area of nonreflecting boundary conditions (NRBCs) for unsteady flow computations. As a part of the development of the space-time conservation element and solution element (CE/SE) method, sets of NRBCs for 1D Euler problems are developed without using any characteristics-based techniques. These conditions are much simpler than those commonly reported in the literature, yet so robust that they are applicable to subsonic, transonic and supersonic flows even in the presence of discontinuities. In addition, the straightforward multidimensional extensions of the presnt 1D NRBCs have been shown numerically to be equally simple and robust. The paper details the theoretical underpinning of these NRBCs, and explains their unique robustness and accuracy in terms of the conservation of space-time fluxes. Some numerical results for an extended Sod's shock-tube problem, illustrating the effectiveness of the present NRBCs are included, together with an associated simple Fortran computer program. As a preliminary to the present development, a review of the basic CE/SE schemes is also included.
\end{abstract}

\section{Introduction}

Because of computing resource limitation and other considerations, it is often required that the computational domain used in a flow simulation represent only a subdomain of a larger physical domain. To obtain a numerical solution that closely resembles the physical flow field in this subdomain, ideally the conditions at the computational boundary should be specified using the physical flow conditions there. Unfortunately, these conditions generally are not known without first solving the larger physical flow field.

Despite the above difficulty, with proper boundary treatments, an accurate simulation of the physical flow over a subdomain using a computational domain that covers only the subdomain is possible if certain conditions are met. As an example, assume that flow disturbances are generated within the subdomain while no disturbances enter it from outside. For this case, an accurate numerical solution over the subdomain can be obtained by imposing proper nonreflecting conditions at the computational boundary. These nonreflecting boundary conditions (NRBCs) are designed such that flow disturbances can propagate out of the computational 
domain smoothly without inducing substantial spurious reflections from the boundary. Such reflections can distort the computed solution and render it completely useless.

Design and application of NRBCs have been a research topic for a long time [18]. In the following, we describe three main established approaches for implemeting NRBCs.

The first approach is based on 1D characteristic decomposition of flow variables and it is most suitable when the waves propagate toward the boundary in the normal direction. Engquist and Majda [1] express the nonreflecting boundary condition as the requirement that the local perturbation propagating along the incoming characteristics be made to vanish. In practice, this consists of projecting the flow equations onto the normal direction of the boundary, converting the conservative variables to characteristic variables, finding the characteristics that enter the domain, and finally setting the corresponding characteristic variables to zero. Although such a procedure is delicate and tedious, it still represents the most commonly used nonreflecting boundary treatment $[2,3,5]$

The second approach is based on the far-field asymptotic solutions [4] and it is ideal when the mean flow near the boundary is nearly uniform.

In the third approach [6-8], efforts are made to insure that the disturbances in the buffer zone that lies outside the computatinal boundary will not reflect back into the computational domain. The most recent development in this area is the so called perfectly matched layer (PML) method [7].

Generally speaking, the established methods described above are not designed for flow problems involving shocks and contact discontinuities. Furthermore, their implementation generally requires a considerable effort.

As an integral part of the development of the space-time conservation element and solution element (CE/SE) method [9-40], several sets of NRBCs for 1D Euler problems will be derived in this paper using a nontraditional concept based entirely on a simple assumption about the space-time flux distribution in the neighborhood of a spatial boundary. As such, the derivation of these NRBCs is consistent with the two basic tenets [15, p. 89] of the CE/SE development, i.e., (i) to capture physics more efficiently and realistically, the modeling focus should be placed on the original integral form of the physical conservation laws, rather than the differential form (which follows from the integral form under the additional smoothness assumption); and (ii) to simplfy mathematics, the use of any elaborate partial differential equation theory should be avoided as much as possible. As will be shown, the derived NRBCs are indeed very simple (e.g., the solution values at a boundary mesh point are simply taken from those at a neighboring interior mesh point), and yet so robust that they are applicable to subsonic, transonic and supersonic flows even in the presence of discontinuities. In addition, as will be described further in Sec. 6, the straightforward multidimensional extensions of the present 1D NRBCs have been shown numerically to be equally effective and robust.

The space-time CE/SE method is a high-resolution and genuinely multidimensional method for solving conservation laws. It is not an incremental improvement of a previously existing method, and it has many nontraditional features. They include: (i) a unified treatment of space and time, (ii) the introduction of conservation elements (CEs) and solution element (SEs) as the vehicles for enforcing space-time flux conservation, and (iii) a time marching strategy that has a spacetime staggered stencil at its core and, as such, can capture shocks without using 
Riemann solvers. Note that conservation elements are nonoverlapping space-time subdomains introduced such that (i) the computational domain can be filled by these subdomains; and (ii) flux conservation can be enforced over each of them and also over the union of any combination of them. On the other hand, solution elements are nonoverlapping space-time subdomains introduced such that (i) the boundary of any CE is covered by a combination of SEs; and (ii) any physical flux vector is approximated using simple smooth functions within a SE. In general, a CE does not coincide with a SE.

Without using preconditioning or other special techniques, since its inception [9] the $\mathrm{CE} / \mathrm{SE}$ method has been used to obtain numerous highly accurate 1D, 2D and 3D steady and unsteady flow solutions with Mach numbers ranging from 0.0028 to 10 . The flow phenomena modeled include traveling and interacting shocks, acoustic waves, shedding vortices, shock/boundary-layer interaction, detonation waves, cavitation and hydraulic jump. In particular, the rather unique capability of the CE/SE method to resolve both strong shocks and small disturbances (e.g., acoustic waves) simultaneously [19,21-26] makes it a unique tool for attacking the problems in computational aeroacustics (CAA). Note that the fact that the (second order) $\mathrm{CE} / \mathrm{SE}$ method can solve CAA problems accurately is an exception to the commonly-held wisdom that a second-order scheme is not adequate for solving CAA problems. Also note that, while numerical dissipation is required for shock capturing, it may also result in annihilation of small disturbances. Thus a solver that can handle both strong shocks and small disturbances simultaneously must be able to overcome this difficulty.

The rest of the paper is organized as follows: A brief description of the CE/SE method is provided in Sec. 2. The concept of generalized conservation elements is introduced in Sec. 3. Using the preliminaries given in Secs. 2 and 3, several sets of NRBCs are derived in Sec. 4. Numerical results that validate the derived NRBCs are given in Sec. 5. The concluding remarks are given in Sec. 6. Finally, to give the reader a concrete example about the simplicity and robustness of the $\mathrm{CE} / \mathrm{SE}$ method in general and the the current NRBCs in particular, a short Fortran program for solving an extended Sod's shock tube problem with a set of NRBCs imposed at its two open ends is listed in Appendix.

\section{Review of the CE/SE method} PDE

As an example, the 1D CE/SE method will be described by considering the

$$
\frac{\partial u}{\partial t}+a \frac{\partial u}{\partial x}=0
$$

where $a$ is a constant. Let $x_{1}=x$, and $x_{2}=t$ be considered as the coordinates of a two-dimensional Euclidean space $E_{2}$. Then, by using Gauss' divergence theorem in the space-time $E_{2}$, it can be shown that Eq. (2.1) is the differential form of the integral conservation law

$$
\oint_{S(V)} \vec{h} \cdot d \vec{s}=0
$$

As depicted in Fig. 1, here (i) $S(V)$ is the boundary of an arbitrary space-time region $V$ in $E_{2}$, (ii) $\vec{h}=(a u, u)$, and (iii) $d \vec{s}=d \sigma \vec{n}$ with $d \sigma$ and $\vec{n}$, respectively, being the area and the unit outward normal of a surface element on $S(V)$. Note 
that: (i) because $\vec{h} \cdot d \vec{s}$ is the space-time flux of $\vec{h}$ leaving the region $V$ through the surface element $d \vec{s}$, Eq. (2.2) simply states that the total space-time flux of $\vec{h}$ leaving $V$ through $S(V)$ vanishes; (ii) in $E_{2}, d \sigma$ is the length of a line segment on the simple closed curve $S(V)$; and (iii) all mathematical operations can be carried out as though $E_{2}$ were an ordinary two-dimensional Euclidean space.

To proceed, let $\Omega$ denote the set of all space-time staggered mesh points $(j, n)$ in $E_{2}$ (dots in Fig. 2(a)), where $n=0, \pm 1 / 2, \pm 1, \pm 3 / 2, \pm 2, \ldots$, and, for each $n$, $j=n \pm 1 / 2, n \pm 3 / 2, n \pm 5 / 2, \ldots$. Each $(j, n) \in \Omega$ is associated with a solution element, i.e., $\mathrm{SE}(j, n)$. By definition, $\mathrm{SE}(j, n)$ is the interior of the space-time region bounded by a dashed curve depicted in Fig. 2(b). It includes a horizontal line segment, a vertical line segment, and their immediate neighborhood.

For any $(x, t) \in \mathrm{SE}(j, n), u(x, t)$ and $\vec{h}(x, t)$, respectively, are approximated by

$$
u^{*}(x, t ; j, n) \stackrel{\text { def }}{=} u_{j}^{n}+\left(u_{x}\right)_{j}^{n}\left(x-x_{j}\right)+\left(u_{t}\right)_{j}^{n}\left(t-t^{n}\right)
$$

and

$$
\vec{h}^{*}(x, t ; j, n) \stackrel{\text { def }}{=}\left(a u^{*}(x, t ; j, n), u^{*}(x, t ; j, n)\right)
$$

Note that (i) $u_{j}^{n},\left(u_{x}\right)_{j}^{n}$, and $\left(u_{t}\right)_{j}^{n}$ are constants in $\operatorname{SE}(j, n)$, (ii) $\left(x_{j}, t^{n}\right)$ are the coordinates of the mesh point $(j, n)$, and (iii) Eq. (2.4) is the numerical analogue of the definition $\vec{h}=(a u, u)$.

Let $u=u^{*}(x, t ; j, n)$ satisfy Eq. (2.1) within $\operatorname{SE}(j, n)$. Then one has $\left(u_{t}\right)_{j}^{n}=$ $-a\left(u_{x}\right)_{j}^{n}$. As a result, Eq. (2.3) reduces to

$$
u^{*}(x, t ; j, n)=u_{j}^{n}+\left(u_{x}\right)_{j}^{n}\left[\left(x-x_{j}\right)-a\left(t-t^{n}\right)\right], \quad(x, t) \in \mathrm{SE}(j, n)
$$

i.e., $u_{j}^{n}$ and $\left(u_{x}\right)_{j}^{n}$ are the only independent marching variables associated with $(j, n)$.

Let $E_{2}$ be divided into nonoverlapping rectangular regions (see Fig. 2(a)). As depicted in Figs. 2(c)-2(e), (i) two such regions, i.e., CE_ $(j, n)$ and $\mathrm{CE}_{+}(j, n)$, are associated with each interior mesh point $(j, n) \in \Omega$; and (ii) $\mathrm{CE}(j, n)$ is the union of $\mathrm{CE}_{-}(j, n)$ and $\mathrm{CE}_{+}(j, n)$.

Note that, among the line segments forming the boundary of $\mathrm{CE}_{-}(j, n), \overline{A B}$ and $\overline{A D}$ belong to $\mathrm{SE}(j, n)$, while $\overline{C B}$ and $\overline{C D}$ belong to $\mathrm{SE}(j-1 / 2, n-1 / 2)$. Similarly, the boundary of $\mathrm{CE}_{+}(j, n)$ belongs to either $\mathrm{SE}(j, n)$ or $\mathrm{SE}(j+1 / 2, n-1 / 2)$. As a result, by imposing two conservation conditions at each $(j, n) \in \Omega$, i.e.,

$$
\oint_{S\left(C E_{+}(j, n)\right)} \vec{h}^{*} \cdot d \vec{s}=0, \quad \text { and } \quad \oint_{S\left(C E_{-}(j, n)\right)} \vec{h}^{*} \cdot d \vec{s}=0, \quad(j, n) \in \Omega
$$

and using Eqs. (2.4) and (2.5), one can obtain two equations for the two unknowns $u_{j}^{n}$ and $\left(u_{x}\right)_{j}^{n}$. By solving these equations, one has (i)

$$
u_{j}^{n}=\frac{1}{2}\left\{(1+\nu) u_{j-1 / 2}^{n-1 / 2}+(1-\nu) u_{j+1 / 2}^{n-1 / 2}+\left(1-\nu^{2}\right)\left[\left(u_{x}^{+}\right)_{j-1 / 2}^{n-1 / 2}-\left(u_{x}^{+}\right)_{j+1 / 2}^{n-1 / 2}\right]\right\}
$$

and, assuming $1-\nu^{2} \neq 0$, (ii)

$$
\left(u_{x}^{+}\right)_{j}^{n}=\frac{1}{2}\left[u_{j+1 / 2}^{n-1 / 2}-u_{j-1 / 2}^{n-1 / 2}-(1-\nu)\left(u_{x}^{+}\right)_{j-1 / 2}^{n-1 / 2}-(1+\nu)\left(u_{x}^{+}\right)_{j+1 / 2}^{n-1 / 2}\right]
$$


Here, (i) $\nu \stackrel{\text { def }}{=} a \Delta t / \Delta x$, and (ii) for any $(j, n) \in \Omega$,

$$
\left(u_{x}^{+}\right)_{j}^{n} \stackrel{\text { def }}{=} \frac{\Delta x}{4}\left(u_{x}\right)_{j}^{n}
$$

Note that derivation of Eqs. (2.7) and (2.8) can be facilitated by the following observations: because $u^{*}(x, t ; j, n)$ is linear in $x$ and $t$, it can be shown that the total flux of $\vec{h}^{*}$ leaving CE_ $(j, n)$ or $\mathrm{CE}_{+}(j, n)$ through any of the four line segments that form its boundary is equal to the scalar product of the vector $\vec{h}^{*}$ evaluated at the midpoint of the line segment and the "surface" vector (i.e., the unit outward normal multiplied by the length) of the line segment.

The $a$ scheme $[9,11,17]$, the explicit nondissipative CE/SE solver for Eq. (2.1), is formed by Eqs. (2.7) and (2.8). Because, for any $(j, n) \in \Omega$, the total flux of $\vec{h}^{*}$ leaving each of $\mathrm{CE}_{-}(j, n)$ and $\mathrm{CE}_{+}(j, n)$ vanishes (see Eq. $\left.(2.6)\right), C E_{-}(j, n)$ and $C E_{+}(j, n),(j, n) \in \Omega$, will be referred to as the conservation elements (CEs) of the a scheme. In addition, because the surface integration over any interface separating two neighboring CEs is evaluated using the information from a single SE, obviously the flux leaving one of these CEs through the interface is the negative of that leaving another $\mathrm{CE}$ through the same interface. As a result, the local conservation relations Eq. (2.6) lead to a global flux conservation relation, i.e., the total flux of $\vec{h}^{*}$ leaving the boundary of any space-time region that is the union of any combination of $C E$ s will also vanish. In particular, because $\mathrm{CE}(j, n)$ is the union of $\mathrm{CE}_{-}(j, n)$ and $\mathrm{CE}_{+}(j, n)$,

$$
\oint_{S(C E(j, n))} \vec{h}^{*} \cdot d \vec{s}=0, \quad(j, n) \in \Omega
$$

must follow from Eq. (2.6). In fact, it can be shown that Eq. (2.10) is equivalent to Eq. (2.7).

In addition to the nondissipative $a$ scheme, there is a family of dissipative $\mathrm{CE} / \mathrm{SE}$ solvers of Eq. (2.1) in which only the less stringent conservation condition Eq. (2.10) is assumed [11]. Because Eq. (2.10) is equivalent to Eq. (2.7), for each of these schemes, $u_{j}^{n}$ is still evaluated using Eq. (2.7) while $\left(u_{x}^{+}\right)_{j}^{n}$ is evaluated in terms of $u_{j \pm 1 / 2}^{n-1 / 2}$ and $\left(u_{x}^{+}\right)_{j \pm 1 / 2}^{n-1 / 2}$ using an equation different from Eq. (2.8). Hereafter any member of this family of dissipative extensions of the $a$ scheme will be referred to as an $a^{\prime}$ scheme. Among the $a^{\prime}$ schemes is one (referred to as the $a-\alpha$ scheme) which is among the simplest and yet capable of handling solutions with discontinuities. For this scheme, $\left(u_{x}^{+}\right)_{j}^{n}$ is evaluated using a finite-difference/weighted-average procedure which involves a parameter $\alpha$ (see Eqs. (2.62), (2.63) and (2.65) in [17]). Note that, because only Eq. (2.10), but not Eq. (2.6), is satisfied by an $a^{\prime}$ scheme, the CEs of an $a^{\prime}$ scheme are $C E(j, n),(j, n) \in \Omega$, rather than $C E_{ \pm}(j, n),(j, n) \in \Omega$.

The $a$ scheme and its extensions described above have been extended to become solvers of systems of conservation laws in one, two and three spatial dimensions [10$12,15,16,20,32]$. In particular, the method was extended to two and three spatial dimensions by using triangles and tetrahedrons, respectively, as the basic building blocks of the spatial meshes $[15,16,32]$. In addition, it was also extended to two and three spatial dimensions by using quadrilaterals and hexahedrons, respectively, as the basic building blocks of the spatial meshes [20]. As a preliminary for Secs. $3-5$, the 1D Euler equations along with the associated CE/SE solvers are briefly discussed in the following. 
The 1D Euler equations of a perfect gas can be expressed as [11]

$$
\frac{\partial u_{m}}{\partial t}+\frac{\partial f_{m}}{\partial x}=0, \quad m=1,2,3
$$

where (i) the conservative flow variables $u_{m}, m=1,2,3$ are functions of the mass density $\rho$, the fluid velocity $v$ and the static pressure $p$; and (ii) $f_{m}, m=1,2,3$, are functions of $u_{m}, m=1,2,3$. The integral form of Eq. (2.11) in space-time $E_{2}$ is

$$
\oint_{S(V)} \vec{h}_{m} \cdot d \vec{s}=0, \quad m=1,2,3
$$

where $\vec{h}_{m}=\left(f_{m}, u_{m}\right), m=1,2,3$, are the space-time mass, momentum, and energy current density vectors, respectively.

The procedures used to construct the $a$ scheme and its extensions was extended to construct several 1D Euler solvers [10-12]. For these Euler solvers, the independent unknowns at each $(j, n) \in \Omega$ are $\left(u_{m}\right)_{j}^{n}$ and $\left(u_{m x}\right)_{j}^{n}, m=1,2,3$. Also, (i) the Euler version of Eq. (2.9) is

$$
\left(u_{m x}^{+}\right)_{j}^{n} \stackrel{\text { def }}{=} \frac{\Delta x}{4}\left(u_{m x}\right)_{j}^{n}
$$

(ii) the 1D Euler version of the $a$ scheme is constructed using the conservation conditions

$$
\oint_{S\left(C E_{+}(j, n)\right)} \vec{h}_{m}^{*} \cdot d \vec{s}=0, \quad \text { and } \quad \oint_{S\left(C E_{-}(j, n)\right)} \vec{h}_{m}^{*} \cdot d \vec{s}=0, \quad m=1,2,3
$$

where $\vec{h}_{m}^{*}$ is the Euler version of $\vec{h}^{*}$; (iii) the 1D Euler version of an $a^{\prime}$ scheme (referred to hereafter as a 1D Euler $a^{\prime}$ scheme) is only required to satisfy the less stringent conservation conditions

$$
\oint_{S(C E(j, n))} \vec{h}_{m}^{*} \cdot d \vec{s}=0, \quad m=1,2,3
$$

and (iv) the 1D Euler version of the $a$ - $\alpha$ scheme (referred to hereafter as the $1 \mathrm{D}$ Euler $a-\alpha$ scheme), which is defined by Eqs. (4.24) and (4.38) in [11], has also been shown to be an accurate and robust shock-capturing solver.

\section{Generalized Conservation Elements}

For any $a^{\prime}$ scheme, $\mathrm{CE}(j, n)$ (see Fig. 2(e)) is a CE. In other words, Eq. (2.10) is valid if, with the aid of Eqs. (2.4), (2.5) and (2.9),

(a) $\vec{h}^{*}$ at any point on $\overline{C B}$ and $\overline{C D}$ is evaluated using $u_{j-1 / 2}^{n-1 / 2}$ and $\left(u_{x}^{+}\right)_{j-1 / 2}^{n-1 / 2}$,

(b) $\vec{h}^{*}$ at any point on $\overline{E D}$ and $\overline{E F}$ is evaluated using $u_{j+1 / 2}^{n-1 / 2}$ and $\left(u_{x}^{+}\right)_{j+1 / 2}^{n-1 / 2}$, and

(c) $\vec{h}^{*}$ at any point on $\overline{A B}$ and $\overline{A F}$ is evaluated using $u_{j}^{n}$ and $\left(u_{x}^{+}\right)_{j}^{n}$.

However, $C E_{+}(j, n)$ and $C E_{-}(j, n)$ (see Figs. 2(c) and 2(d)) are not $C E$ s of an $a^{\prime}$ scheme. In other words, Eq. (2.6) is not valid if

(d) $\vec{h}^{*}$ at any point on $\overline{A D}$ (which is a part of $\mathrm{SE}(j, n)$ ) is evaluated using $u_{j}^{n}$ and $\left(u_{x}^{+}\right)_{j}^{n}$.

Assume rules (a)-(c). Then the above discussion implies that, for an $a^{\prime}$ scheme, the total flux of $\vec{h}^{*}$ leaving the boundary of $\mathrm{CE}_{-}(j, n)$ vanishes only if we ignore the rule (d) and instead assume the rule: 
(e) the flux leaving $\mathrm{CE}_{-}(j, n)$ through $\overline{A D}$ is defined to be the value such that the total flux leaving the boundary of $\mathrm{CE}_{-}(j, n)$ vanishes.

Note that, unlike the rule (d), the new rule (e) does not specify $\vec{h}^{*}$ at each individual point on $\overline{A D}$. The rule (e) only defines a scalar, i.e., the total flux passing through the entire line segment $\overline{A D}$. Because the flux-conservation property of $\mathrm{CE}_{-}(j, n)$ is established by imposing the rule (e), a space-time region such as $\mathrm{CE}_{-}(j, n)$ will be referred to as a generalized conservation element (GCE).

To proceed, without exception hereafter the flux leaving a space-time region $A$ through an interface separating $A$ and a neighboring region $B$ is assumed to be the same flux as that entering $B$ through the interface. As an example, the flux leaving CE_ $(j, n)$ through $\overline{A D}$ is the same flux that entering $\mathrm{CE}_{+}(j, n)$ through $\overline{A D}$ (i.e., the flux leaving $\mathrm{CE}_{-}(j, n)$ through $\overline{A D}$ is the negative of the flux that leaving $\mathrm{CE}_{+}(j, n)$ through $\left.\overline{A D}\right)$. Then because (i) $\mathrm{CE}(j, n)$ is the union of CE_ $(j, n)$ and $\mathrm{CE}_{+}(j, n)$, and (ii) $\mathrm{CE}(j, n)$ is a $\mathrm{CE}$ for any $a^{\prime}$ scheme, the rules (a)-(c) and (e) lead to the conclusion that $\mathrm{CE}_{+}(j, n)$ is also a GCE for any $a^{\prime}$ scheme. In fact, assuming the rules (a)-(c), the flux at $\overline{A D}$ defined by the rule (e) is identical to that defined by the rule:

(f) the flux leaving $\mathrm{CE}_{+}(j, n)$ through $\overline{A D}$ is defined to be the value such that the total flux leaving the boundary of $\mathrm{CE}_{+}(j, n)$ vanishes.

At this juncture, note that the concept of GCEs is introduced such that the physical conservation law Eq. (2.2) can still have a numerical analogue in a space-time region that is a subset of a $C E$ of any $a^{\prime}$ scheme.

By using a general rule that is similar to the rule (e), we can define other GCEs for any $a^{\prime}$ scheme. As an example, consider Fig. 3(a). Here $P$ denotes any point on $\overline{B C}$ ( $P$ may coincide with point $B$ or point $C$ ). Because the fluxes leaving the triangle $A B P$ through $\overline{B P}$ and $\overline{A B}$, respectively, can be evaluated using the rules (a) and (c), one can define the flux leaving $A B P$ through $\overline{A P}$ to be the value such that the total flux leaving the boundary of $A B P$ vanishes. With this definition, $A B P$ becomes a GCE. Because the quadrilateral $A B C D$, (i.e., $\mathrm{CE}_{-}(j, n)$ ) is a GCE, using an argument similar to that was used to establish the equivalence of the above rules (e) and (f), one concludes that the quadrilateral $A P C D$ is also a GCE. To emphasize the fact that $\vec{h}^{*}$ at each individual point on either $\overline{A D}$ or $\overline{A P}$ is not specified, $\overline{A D}$ and $\overline{A P}$ are denoted by dashed lines in Fig. 3(a). The same convention will also be used in the rest of this paper.

In a similar fashion, one can divide $\mathrm{CE}_{-}(j, n)$ or $\mathrm{CE}_{+}(j, n)$ into other combinations of two GCEs (see Fig. 3(b)).

Furthermore, by (i) dividing the quadrilateral $A P C D$ (a GCE) in Fig. 3(a) into two triangles $A P D$ and $D P C$ (see Fig. 3(c)), and (ii) defining the flux leaving $D P C$ through $\overline{D P}$ to be the value such that the total flux leaving the boundary of $D P C$ vanishes, one can divide $\mathrm{CE}_{-}(j, n)$ into three GCEs, i.e., the triangles $A B P$, $A P D$ and $D P C$. In a similar fashion, one can divide $\mathrm{CE}_{-}(j, n)$ or $\mathrm{CE}_{+}(j, n)$ into other combinations of three or more GCEs. The discussion of GCEs for any $a^{\prime}$ scheme is concluded with the following remarks:

(a) Consider Fig. 4. Let $P$ be an interior point on $\overline{A D}$. Because the rule (e) does not specify the flux passing through $\overline{A P}$ or $\overline{P D}$, one may not assign a flux on $\overline{C P}$ such that the triangle $P C D$ and the quadrilateral $A B C P$ can be considered as GCEs. 
(b) Consider Fig. 5(a). Let $\vec{h}^{*}$ be given at every point on $\overline{B C}, \overline{C D}, \overline{D G}$, and $\overline{G F}$ (these line segments are denoted by solid lines). As a result, the flux on $\overline{B C}$, $\overline{C E}, \overline{E A}, \overline{A D}, \overline{D G}$ and $\overline{G F}$ are also given. It follows that one can assign a flux on $\overline{A B}$ and also a flux on $\overline{E F}$ such that both the triangle $A B C$ and the quadrilateral $D E F G$ are GCEs. Because the fluxes at all line segments that form the boundary of the union of $A B C$ and $D E F G$ are well defined and the total flux leaving the boundary vanishes, the union is also a GCE.

(c) Consider Fig. 5(b). This figure is identical to Fig. 5(a) except that $\vec{h}^{*}$ is now given at every point on $\overline{A B}, \overline{B C}, \overline{D G}, \overline{G F}$, and $\overline{F E}$. As a result, one can assign a flux on $\overline{A C}$ and also a flux on $\overline{D E}$ such that both $A B C$ and $D E F G$ are GCEs. However, the question of whether the union of $A B C$ and $D E F G$ is a GCE is an ill-posed problem because (i) the flux at $\overline{C E}$ and that at $\overline{A D}$ are not uniquely defined, and (ii) $\overline{C E}$ and $\overline{A D}$ form part of the boundary of this union.

Finally, note that the discussions of GCEs given above are applicable to any Euler version of any $a^{\prime}$ scheme if $\vec{h}^{*}$ is replaced by each $\vec{h}_{m}^{*}, m=1,2,3$. This fact becomes apparent if one recalls that Eq. (2.15) is satisfied by any Euler version of any $a^{\prime}$ scheme, and also observes that Eq. (2.15), for each $m=1,2,3$, becomes Eq. (2.10) if $\vec{h}_{m}^{*}$ is replaced by $\vec{h}^{*}$.

\section{Nonreflecting Boundary Conditions}

In this section, several sets of NRBCs for the CE/SE method will be derived using a nontraditional approach. As a prerequisite, a set of basic concepts will first be elaborated using a simple initial-value problem as an example.

4.1. Basic Concepts. Consider the initial-value problem defined by Eq. (2.1) and the initial conditions: at $t=0$,

$$
u=\left\{\begin{array}{lll}
U, & \text { if } & x>0 \\
V, & \text { if } & x<0
\end{array}\right.
$$

where $U$ and $V$ are two different given constants. For $t \geq 0$, the exact weak solution to this problem is

$$
u=\left\{\begin{array}{lll}
U, & \text { if } & x-a t>0 \\
V, & \text { if } & x-a t<0
\end{array}\right.
$$

Obviously, the space-time variation of the above solution is solely driven by the initial-data discontinuity that occurs at $x=0$.

To construct a corresponding discretized initial-value problem, consider an unbounded and uniform space-time mesh formed by the mesh points $(j, n) \in \Omega$ with $n \geq 0$ (see Fig. 6). The numerical analogue of the initial conditions Eq. (4.1) can be expressed as (i)

$$
u_{j}^{0}=\left\{\begin{array}{lll}
U, & \text { if } j=1 / 2,3 / 2, \ldots \\
V, & \text { if } j=-1 / 2,-3 / 2, \ldots
\end{array}\right.
$$

and (ii)

$$
\left(u_{x}^{+}\right)_{j}^{0}=0, \quad \text { for } \quad j= \pm 1 / 2, \pm 3 / 2, \ldots
$$

Let $\Omega_{+}$be the subset of $\Omega$ with $n>0$. Then, for any $(j, n) \in \Omega_{+}, u_{j}^{n}$ and $\left(u_{x}^{+}\right)_{j}^{n}$ can be determined in terms of the above given initial data through the marching of 
a given $a^{\prime}$ scheme. Hereafter the solution thus obtained and the associated initialvalue problem will be referred to as Solution I and Problem I, respectively. As in the case of its analytical counterpart, the space-time variation of Solution I is also solely driven by the initial-data discontinuity that occurs across the mesh interval centered at $x=0$.

The mesh depicted in Fig. 6 is unbounded. In reality one can only use a bounded mesh. To proceed, first we shall define several finite subsets of $\Omega_{+}$. Let (i) $j_{0} \gg 1$ and $n_{0}>0$ be given whole integers, and (ii) $j_{b} \stackrel{\text { def }}{=} j_{0}+1 / 2$. Then the mesh-point set $\Lambda_{+}\left(j_{0}, n_{0}\right)$ and its subsets $\Lambda_{+}^{\prime}\left(j_{0}, n_{0}\right)$ and $\partial \Lambda_{+}\left(j_{0}, n_{0}\right)$ are defined as follows: (i) $(j, n) \in \Lambda_{+}\left(j_{0}, n_{0}\right)$ if and only if $(j, n) \in \Omega_{+},|j| \leq j_{b}$ and $n \leq n_{0}+1 / 2$ (i.e., $\Lambda_{+}\left(j_{0}, n_{0}\right)$ is the set of the mesh points $(j, n)$ depicted in Fig. 7 excluding those with $n=0)$; (ii) $(j, n) \in \Lambda_{+}^{\prime}\left(j_{0}, n_{0}\right)$ if and only if $(j, n) \in \Lambda_{+}\left(j_{0}, n_{0}\right)$ and $|j|<j_{b}$, and (iii) $(j, n) \in \partial \Lambda_{+}\left(j_{0}, n_{0}\right)$ if and only if $(j, n) \in \Lambda_{+}\left(j_{0}, n_{0}\right)$ and $|j|=j_{b}$. Obviously $\Lambda_{+}\left(j_{0}, n_{0}\right)=\Lambda_{+}^{\prime}\left(j_{0}, n_{0}\right) \cup \partial \Lambda_{+}\left(j_{0}, n_{0}\right)$.

Next we cosider the initial data defined by (i)

$$
u_{j}^{0}=\left\{\begin{array}{lll}
U, & \text { if } j=1 / 2,3 / 2, \ldots, j_{b} \\
V, & \text { if } j=-1 / 2,-3 / 2, \ldots,-j_{b}
\end{array}\right.
$$

and (ii)

$$
\left(u_{x}^{+}\right)_{j}^{0}=0, \quad \text { for } \quad j= \pm 1 / 2, \pm 3 / 2, \ldots, \pm j_{b}
$$

Note that $u_{j}^{0}$ and $\left(u_{x}^{+}\right)_{j}^{0}$ defined in Eqs. (4.5) and (4.6) are identical to those defined in Eqs. (4.3) and (4.4), respectively, in their common domain defined by $|j| \leq j_{b}$. The question arises that, given only the truncated initial data Eqs. (4.5) and (4.6), is it still possible to obtain a solution in $\Lambda_{+}^{\prime}\left(j_{0}, n_{0}\right)$ that is reasonably close to Solution I?

To answer the above question, first consider a discrete initial/boundary-value problem in which the initial data are specified using Eqs. (4.5) and (4.6), and the boundary data $u_{j}^{n}$ and $\left(u_{x}^{+}\right)_{j}^{n}$ at all $(j, n) \in \partial \Lambda_{+}\left(j_{0}, n_{0}\right)$ are also given. Because, for any $(j, n) \in \Omega,(j, n)$ and $(j \pm 1 / 2, n-1 / 2)$ form the stencil of the $a^{\prime}$ scheme, one concludes that, for all $(j, n) \in \Lambda_{+}^{\prime}\left(j_{0}, n_{0}\right), u_{j}^{n}$ and $\left(u_{x}^{+}\right)_{j}^{n}$ can be uniquely determined in terms of the above given initial and boundary data by using the same $a^{\prime}$ scheme that was used to generate Solution I. Hereafter, the new solution thus obtained and the associated initial/boundary-value problem will be referred to as Solution II and Problem II, respectively. Obviously, Solution II is dependent on the boundary data specified on $\partial \Lambda_{+}\left(j_{0}, n_{0}\right)$. By definition, the degree of non-reflectiveness of the boundary conditions of Problem II will be measured by how closely Solutions I and II are matched in $\Lambda_{+}^{\prime}\left(j_{0}, n_{0}\right)$. In other words, the closer the match the less reflective these boundary conditions are. In particular, they are said to be perfectly nonreflecting if the match is perfect.

Next note that, again because of the shape of the stencil of an $a^{\prime}$ scheme described earlier, for Solution I, $u_{j}^{n}$ and $\left(u_{x}^{+}\right)_{j}^{n}$ at all $(j, n) \in \Lambda_{+}\left(j_{0}, n_{0}\right)$ are completed determined by the initial data given at the mesh points $(j, 0), j= \pm 1 / 2, \pm 3 / 2, \ldots$, $\pm\left(j_{b}+n_{0}\right)$ (see Figs. 6 and 7$)$. As a result, one can see easily that Solutions I and II must be identical in $\Lambda_{+}^{\prime}\left(j_{0}, n_{0}\right)$ if the values of Solution I at $\partial \Lambda_{+}\left(j_{0}, n_{0}\right)$ are used as the boundary data for Problem II. In other words, the boundary conditions formed using these boundary data are perfectly nonreflecting for Problem II. Unfortunately, obtaining these boundary data requires solving a problem with a larger spatial domain, whose size increases as $n_{0}$ increases. 
The key propositions that emerge from the above discussions are:

(a) Any set of NRBCs for Problem II must meet the requirement that the resulting Solution II more or less matches Solution I in $\Lambda_{+}^{\prime}\left(j_{0}, n_{0}\right)$.

(b) In constructing any set of NRBCs for Problem II, only the information extracted from the initial data Eqs. (4.5) and (4.6) can be used.

(c) The values of Solution I in $\Lambda_{+}^{\prime}\left(j_{0}, n_{0}\right)$ generally are dependent on some initial data beyond those given in Eqs. (4.5) and (4.6).

It follows from these propositions that construction of NRBCs for Problem II is possible only if the contribution to Solution I in $\Lambda_{+}^{\prime}\left(j_{0}, n_{0}\right)$ by the initial data $u_{j}^{0}$ and $\left(u_{x}^{+}\right)_{j}^{0}$ with $|j|>j_{b}$ somehow can be extrapolated based on the truncated initial data Eq. (4.5) and (4.6). Fortunately, the existence of such an extrapolation is supported by the following facts:

(a) Except in the neighborhood $|j| \leq 1 / 2$, the initial data Eqs. (4.5) and (4.6) do not vary in each of the remaining regions $1 / 2 \leq j \leq j_{b}$ and $-j_{b} \leq j \leq-1 / 2$.

(b) Not only does the initial data Eqs. (4.3) and (4.4) coincide with those of Eqs. (4.5) and (4.6) in their common region $|j| \leq j_{b}$, in the region $j>j_{b}$ $\left(j<-j_{b}\right)$, the former initial data also have the same constant values of those in the region $1 / 2 \leq j \leq j_{b}\left(-j_{b} \leq j \leq-1 / 2\right)$.

In the following, it will be shown that practical, albeit not perfect, nonreflecting boundary conditions for Problem II can be constructed using an assumption about the flux distribution near its spatial boundaries. The exact meaning of this assumption will become completely clear only after its role in constructing NRBCs is shown explicitly. However the gist of this assumption will be described immediately.

As an example, consider the mesh line $j=j_{b}$, i.e., the right boundary mesh line of Problem II depicted in Figs. 6 and 7. Given Solution I, one can evaluate, from $t=0$ to $t=n_{0} \Delta t$, the distribution of the space-time flux of $\vec{h}^{*}$ passing through (say the positive direction is from left to right) the above mesh line and its neighboring vertical mesh lines. Because the space-time variation of Solution I is solely driven by an initial-data discontinuity that is located to the left of the mesh line $j=j_{b}$, no propagating "solution disturbances" will ever reach this mesh line from its right side. As a result, one may expect that, for the time interval $\left(0, n_{0} \Delta t\right)$, the flux distribution of Solution $I$ at the mesh line $j=j_{b}$ is not "dependent" on those at the neighboring vertical mesh lines with $j>j_{b}$, i.e., it is "dependent" only on those at the neighboring vertical mesh lines with $j<j_{b}$. In fact one may further assume that, for the time interval $\left(0, n_{0} \Delta t\right)$, the flux distribution of Solution $I$ at the mesh line $j=j_{b}$ is a smooth extrapolation of those at the neighboring mesh lines with $j<j_{b}$. Because a set of NRBCs for Problem II is introduced such that the resulting Solution II more or less matches Solution I in $\Lambda_{+}^{\prime}\left(j_{0}, n_{0}\right)$, it follows that Solution II must possess the same flux extrapolation relation referred to above if the boundary conditions imposed at the mesh line $j=j_{b}$ are indeed nonreflecting. As will be shown shortly, the current NRBCs are derived assuming this flux extrapolation relation. Note that, according to the current line of thinking, a set of NRBCs is one that allows for the flux to smoothly "stream" out of the spatial boundary from the interior.

To elaborate further, consider a 1D problem $(+\infty>x>-\infty)$ that is governed by Eq. (2.1). For such a problem, solution disturbances propagate to the right (left) with a constant speed if $a>0(a<0)$. As a result, no solution disturbances will ever reach the mesh line $j=j_{b}$ from its right side if the initial sources of 
disturbance are located to the left of the mesh line. In contrast, for a more complex problem such as that involving a 1D subsonic Euler flow, (i) disturbances emitted from any source, relative to the stationary computational frame, can propagate in both the right and left directions; and (ii) any disturbance itself is a new source of disturbance. As a result, for such a flow, solution disturbances that originate from a source that is located to the left of the mesh line $j=j_{b}$ can propagate into the region to the right of the mesh line and from there propagate back to the same mesh line. In the current construction of NRBCs, the effect of this "back scattering" phenomenon on the flux distribution along the mesh line $j=j_{b}$ is assumed to be negligible. Generally speaking, the last assumption is valid if the mesh point $\left(0, j_{b}\right)$ is located at a large distance from the nearest initial source of disturbance (such as in the current case in which by assumption $j_{b} \gg 1$, and the initial source of disturbance is located around $j=0$ ).

This subsection is concluded with the following remarks:

(a) The above discussions make it clear that, strictly speaking, NRBCs for a discretized initial/boundary-value problem are not well-defined unless its solution can be compared against that of another problem with a spacetime domain containing that of the original problem.

(b) Conceptually, the reflecting boundary conditions that are imposed on a solid wall are exactly opposite to the NRBCs discussed above. Obviously, the mass flux cannot smoothly "stream" into a solid wall.

(c) In the following subsections, the concept of GCEs introduced in Sec. 3 will be used in the construction of several sets of NRBCs which are applicable to any $a^{\prime}$ scheme. Before proceeding to construct these conditions, the reader again is reminded of the convention that any part of the boundary of a GCE on which $\vec{h}^{*}$ is not specified at each individual point is denoted by a dashed line (see Fig. 4).

4.2. The First and Second Sets of NRBCs. The boundary mesh lines with $j= \pm j_{b}$ that appear in Figs. 6 and 7 along with some of their immediately adjacent interior mesh lines are depicted in Fig. 8. In this figure, the points of intersection between these vertical mesh lines and any horizontal $n$th time-level line, respectively, are denoted by $A_{\ell}, B_{\ell}, C_{\ell}, D_{\ell}, A_{\ell}^{\prime}, B_{\ell}^{\prime}, C_{\ell}^{\prime}$ and $D_{\ell}^{\prime}$, where $\ell=2 n$. Furthermore, for each $\ell=0,1,2, \ldots$, points $P_{\ell}, Q_{\ell}, P_{\ell}^{\prime}$ and $Q_{\ell}^{\prime}$, respectively, are on the line segments $\overline{B_{\ell} C_{\ell}}, \overline{C_{\ell} D_{\ell}}, \overline{B_{\ell}^{\prime} C_{\ell}^{\prime}}$ and $\overline{C_{\ell}^{\prime} D_{\ell}^{\prime}}$ with

$$
\left|\overline{P_{\ell} C_{\ell}}\right|=\lambda \frac{\Delta x}{2}, \quad\left|\overline{C_{\ell} Q_{\ell}}\right|=(1-\lambda) \frac{\Delta x}{2}, \quad\left|\overline{P_{\ell}^{\prime} C_{\ell}^{\prime}}\right|=\lambda^{\prime} \frac{\Delta x}{2}, \quad\left|\overline{C_{\ell}^{\prime} Q_{\ell}^{\prime}}\right|=\left(1-\lambda^{\prime}\right) \frac{\Delta x}{2}
$$

Here $\lambda$ and $\lambda^{\prime}$ are adjustable parameters with $0 \leq \lambda, \lambda^{\prime} \leq 1$. Obviously $\left|\overline{P_{\ell} Q_{\ell}}\right|=$ $\left|\overline{P_{\ell}^{\prime} Q_{\ell}^{\prime}}\right|=\Delta x / 2$. Furthermore, because $\left|\overline{B_{\ell} C_{\ell}}\right|=\left|\overline{C_{\ell} D_{\ell}}\right|=\Delta x / 2$, one concludes that, for $\ell=0,1,2, \ldots$, points $P_{\ell}$ and $Q_{\ell}$, respectively, coincide with (i) points $B_{\ell}$ and $C_{\ell}$ if $\lambda=1$, and (ii) points $C_{\ell}$ and $D_{\ell}$ if $\lambda=0$. As a result, as long as $0 \leq \lambda \leq 1$, for each $\ell=0,2,4, \ldots$, the interior of $\overline{P_{\ell} Q_{\ell}}$ lies entirely within the solution element associated with point $C_{\ell}$. Obviously the above conclusions also hold if points $P_{\ell}, Q_{\ell}, B_{\ell}, C_{\ell}$ and $D_{\ell}$, and the parameters $\lambda$ are replaced by points $P_{\ell}^{\prime}, Q_{\ell}^{\prime}, B_{\ell}^{\prime}, C_{\ell}^{\prime}$ and $D_{\ell}^{\prime}$, and the parameter $\lambda^{\prime}$, respectively.

According to the discussions given in Sec. 3, for any $\ell=0,1,2, \ldots$, the rectangles $A_{\ell} A_{\ell+1} B_{\ell+1} B_{\ell}, P_{\ell} P_{\ell+1} C_{\ell+1} C_{\ell}, C_{\ell} C_{\ell+1} Q_{\ell+1} Q_{\ell}, A_{\ell}^{\prime} A_{\ell+1}^{\prime} B_{\ell+1}^{\prime} B_{\ell}^{\prime}, P_{\ell}^{\prime} P_{\ell+1}^{\prime} C_{\ell+1}^{\prime} C_{\ell}^{\prime}$ 
and $C_{\ell}^{\prime} C_{\ell+1}^{\prime} Q_{\ell+1}^{\prime} Q_{\ell}^{\prime}$ can be considered as GCEs. As a result, the union of any combinations of these GCEs is also a GCE. As an example, for any $\ell=0,2,4, \ldots$, the rectangle $A_{\ell} A_{\ell+2} B_{\ell+2} B_{\ell}$ is a GCE. To proceed, for any $\ell=0,2,4, \ldots$, let (i) $F\left(\overline{A_{\ell} A_{\ell+2}}\right)$ denote the flux of $\vec{h}^{*}$ entering the vertical line segment $\overline{A_{\ell} A_{\ell+2}}$ from the left, and (ii) $F\left(\overline{A_{\ell} B_{\ell}}\right)$ denote the flux of $\vec{h}^{*}$ entering the horizontal line segment $\overline{A_{\ell} B_{\ell}}$ from below. Hereafter, similar notations will be used to denote the fluxes passing through other vertical and horizontal line segments. The simplest NRBCs at the boundary mesh line $j=j_{b}$ will be constructed by assuming the simplest flux extrapolation condition, i.e.,

$$
F\left(\overline{A_{\ell} A_{\ell+2}}\right)=F\left(\overline{B_{\ell} B_{\ell+2}}\right), \quad \ell=0,2,4, \ldots
$$

Because the rectangle $A_{\ell} A_{\ell+2} B_{\ell+2} B_{\ell}$ is a GCE, we have

$$
F\left(\overline{A_{\ell} A_{\ell+2}}\right)-F\left(\overline{B_{\ell} B_{\ell+2}}\right)+F\left(\overline{A_{\ell+2} B_{\ell+2}}\right)-F\left(\overline{A_{\ell} B_{\ell}}\right)=0, \quad \ell=0,2,4, \ldots
$$

Combining Eqs. (4.8) and (4.9), one concludes that

$$
F\left(\overline{A_{\ell+2} B_{\ell+2}}\right)=F\left(\overline{A_{\ell} B_{\ell}}\right), \quad \ell=0,2,4, \ldots
$$

As a result,

$$
F\left(\overline{A_{\ell} B_{\ell}}\right)=F\left(\overline{A_{0} B_{0}}\right), \quad \ell=2,4,6, \ldots
$$

With the aid of Eqs. (2.4), (2.5) and (2.9), and recalling that the length of each $\overline{A_{\ell} B_{\ell}}$ is $\Delta x / 2$, it can be shown that Eq. (4.11) is equivalent to

$$
u_{A_{\ell}}-\left(u_{x}^{+}\right)_{A_{\ell}}=u_{A_{0}}-\left(u_{x}^{+}\right)_{A_{0}}, \quad \ell=2,4,6 \ldots
$$

where $u_{A_{\ell}}$ and $\left(u_{x}^{+}\right)_{A_{\ell}}, \ell=0,2,4, \ldots$, denote the mesh values of $u$ and $u_{x}^{+}$at point $A_{\ell}$, respectively. Note that: (i) hereafter similar notations will be used to denote the mesh values of $u$ and $u_{x}^{+}$at other mesh points; and (ii) according to Eq. (2.9), $\left(u_{x}^{+}\right)_{A_{\ell}}$ and $\left(u_{x}^{+}\right)_{A_{0}}$ are quantities of first-order in $\Delta x$. Also note that, by using a similar approach, it can be shown that the counterpart to Eq. (4.12) for the left boundary is

$$
u_{A_{\ell}^{\prime}}+\left(u_{x}^{+}\right)_{A_{\ell}^{\prime}}=u_{A_{0}^{\prime}}+\left(u_{x}^{+}\right)_{A_{0}^{\prime}}, \quad \ell=2,4,6, \ldots
$$

Let the initial data $u_{A_{0}}$ and $\left(u_{x}^{+}\right)_{A_{0}}$ be given. Then, for each $\ell$, there are only two unknowns, i.e., the boundary data $u_{A_{\ell}}$ and $\left(u_{x}^{+}\right)_{A_{\ell}}$, in Eq. (4.12). As a result, after imposing Eq. (4.12), there is still one degree of freedom left in choosing the two unknowns. To obtain the simplest boundary conditions, the degree of freedom is removed by further assuming that the zero-order term and the first-order term on the left side of the equality sign in Eq. (4.12) are equal to those on the right side, respectively. Thus one has

$$
u_{A_{\ell}}=u_{A_{0}} \quad \text { and } \quad\left(u_{x}^{+}\right)_{A_{\ell}}=\left(u_{x}^{+}\right)_{A_{0}} \quad \ell=2,4,6, \ldots
$$

Similarly, the counterpart to Eq. (4.14) for the left boundary is

$$
u_{A_{\ell}^{\prime}}=u_{A_{0}^{\prime}} \quad \text { and } \quad\left(u_{x}^{+}\right)_{A_{\ell}^{\prime}}=\left(u_{x}^{+}\right)_{A_{0}^{\prime}} \quad \ell=2,4,6 \ldots
$$

Note that, even though it is counter-intuitive, it will be shown in Sec. 5 that the Euler counterparts of the steady-state boundary conditions Eqs. (4.14) and (4.15) turn out to be a set of reasonably good NRBCs. Hereafter Eqs. (4.14) and (4.15) will be referred to as the first set of NRBCs. 
A set of more advanced NRBCs will be constructed assuming

$$
F\left(\overline{A_{\ell} A_{\ell+2}}\right)-F\left(\overline{B_{\ell} B_{\ell+2}}\right)=F\left(\overline{P_{\ell} P_{\ell+2}}\right)-F\left(\overline{Q_{\ell} Q_{\ell+2}}\right), \quad \ell=0,2,4, \ldots
$$

Note that, because (i) the line segments $\overline{A_{\ell} A_{\ell+2}}, \overline{B_{\ell} B_{\ell+2}}, \overline{P_{\ell} P_{\ell+2}}$, and $\overline{Q_{\ell} Q_{\ell+2}}$ have the same length (i.e., $\Delta t$ ), and (ii) the horizontal distance (i.e., $\Delta x / 2$ ) that separates the line segment $\overline{A_{\ell} A_{\ell+2}}$ and $\overline{B_{\ell} B_{\ell+2}}$ is identical to that which separates the line segments $\overline{P_{\ell} P_{\ell+2}}$ and $\overline{Q_{\ell} Q_{\ell+2}}$, Eq. (4.16) represents a linear flux extrapolation relation. Also, because the rectangles $A_{\ell} A_{\ell+2} B_{\ell+2} B_{\ell}$ and $P_{\ell} P_{\ell+2} Q_{\ell+2} Q_{\ell}$ are GCEs, we have Eq. (4.9) and

$$
F\left(\overline{P_{\ell} P_{\ell+2}}\right)-F\left(\overline{Q_{\ell} Q_{\ell+2}}\right)+F\left(\overline{P_{\ell+2} Q_{\ell+2}}\right)-F\left(\overline{P_{\ell} Q_{\ell}}\right)=0
$$

for $\ell=0,2,4, \ldots$ With the aid of Eqs. (4.9) and (4.17), Eq. (4.16) implies that

$$
F\left(\overline{A_{\ell+2} B_{\ell+2}}\right)-F\left(\overline{P_{\ell+2} Q_{\ell+2}}\right)=F\left(\overline{A_{\ell} B_{\ell}}\right)-F\left(\overline{P_{\ell} Q_{\ell}}\right), \quad \ell=0,2,4, \ldots
$$

As a result,

$$
F\left(\overline{A_{\ell} B_{\ell}}\right)-F\left(\overline{P_{\ell} Q_{\ell}}\right)=F\left(\overline{A_{0} B_{0}}\right)-F\left(\overline{P_{0} Q_{0}}\right), \quad \ell=2,4,6, \ldots
$$

With the aid of Eqs. (2.4), (2.5), (2.9) and (4.7), it can be shown that Eq. (4.19) is equivalent to

$$
\begin{aligned}
& \left(u_{A_{\ell}}-u_{C_{\ell}}\right)-\left[\left(u_{x}^{+}\right)_{A_{\ell}}+(2 \lambda-1)\left(u_{x}^{+}\right)_{C_{\ell}}\right] \\
= & \left(u_{A_{0}}-u_{C_{0}}\right)-\left[\left(u_{x}^{+}\right)_{A_{0}}+(2 \lambda-1)\left(u_{x}^{+}\right)_{C_{0}}\right]
\end{aligned}
$$

where $\ell=2,4,6, \ldots$. Similarly, the counterpart to Eq. (4.20) for the left boundary is

$$
\begin{aligned}
& \left(u_{A_{\ell}^{\prime}}-u_{C_{\ell}^{\prime}}\right)+\left[\left(u_{x}^{+}\right)_{A_{\ell}^{\prime}}+\left(2 \lambda^{\prime}-1\right)\left(u_{x}^{+}\right)_{C_{\ell}^{\prime}}\right] \\
= & \left(u_{A_{0}^{\prime}}-u_{C_{0}^{\prime}}\right)+\left[\left(u_{x}^{+}\right)_{A_{0}^{\prime}}+\left(2 \lambda^{\prime}-1\right)\left(u_{x}^{+}\right)_{C_{0}^{\prime}}\right]
\end{aligned}
$$

where $\ell=2,4,6, \ldots$. To proceed, note that Eqs. (4.5) and (4.6) coupled with the assumption $j_{b} \gg 1$ imply that $u_{A_{0}}=u_{C_{0}}=U, u_{A_{0}^{\prime}}=u_{C_{0}^{\prime}}=V$ and $\left(u_{x}^{+}\right)_{A_{0}}=$ $\left(u_{x}^{+}\right)_{C_{0}}=\left(u_{x}^{+}\right)_{A_{0}^{\prime}}=\left(u_{x}^{+}\right)_{C_{0}^{\prime}}=0$. As a result, the expressions on the right sides of Eqs. (4.20) and (4.21) vanish.

Let the parameter $\lambda$ be given. Then because the $C_{\ell}$ is an interior mesh point at the $(\ell / 2)$ th time level (recall that $n=\ell / 2), u_{C_{\ell}}$ and $\left(u_{x}^{+}\right)_{C_{\ell}}$ can be evaluated using the known marching variables at the $((\ell-1) / 2)$ th time level. Thus one concludes that, for each $\ell$, there are only two unknowns, i.e., the boundary data $u_{A_{\ell}}$ and $\left(u_{x}^{+}\right)_{A_{\ell}}$ in Eq. (4.20). As a result, after imposing Eq. (4.20), there is still one degree of freedom left in choosing the two unknowns. To obtain the simplest NRBCs possible, the degree of freedom is removed by further assuming that the sum of the zero-order terms and that of the first-order terms on the left side of Eq. (4.20), respectively, are equal to zero. Thus one has

$$
u_{A_{\ell}}=u_{C_{\ell}} \quad \text { and } \quad\left(u_{x}^{+}\right)_{A_{\ell}}=(1-2 \lambda)\left(u_{x}^{+}\right)_{C_{\ell}} \quad \ell=2,4,6, \ldots
$$

Similarly, the counterpart to Eq. (4.22) for the left boundary is

$$
u_{A_{\ell}^{\prime}}=u_{C_{\ell}^{\prime}} \text { and }\left(u_{x}^{+}\right)_{A_{\ell}^{\prime}}=\left(1-2 \lambda^{\prime}\right)\left(u_{x}^{+}\right)_{C_{\ell}^{\prime}} \quad \ell=2,4,6, \ldots
$$

Note that, because $1 \geq \lambda, \lambda^{\prime} \geq 0$,

$$
1 \geq(1-2 \lambda),\left(1-2 \lambda^{\prime}\right) \geq-1
$$

Hereafter Eqs. (4.22) and (4.23) will be referred to as the second set of NRBCs. 
4.3. The Third Set of NRBCs. The points $A_{\ell}, B_{\ell}, C_{\ell}, A_{\ell}^{\prime}, B_{\ell}^{\prime}$ and $C_{\ell}^{\prime}$, $\ell=0,1,2, \ldots$, that are depicted in Fig. 9 are identical to those depicted in Fig. 8 . Furthermore, for each $\ell=0,1,2, \ldots$, points $R_{\ell}, S_{\ell}, R_{\ell}^{\prime}$ and $S_{\ell}^{\prime}$, respectively, are on the line segments $\overline{A_{\ell} B_{\ell}}, \overline{B_{\ell} C_{\ell}}, \overline{A_{\ell}^{\prime} B_{\ell}^{\prime}}$ and $\overline{B_{\ell}^{\prime} C_{\ell}^{\prime}}$ with

$$
\left|\overline{R_{\ell} B_{\ell}}\right|=\lambda \frac{\Delta x}{2}, \quad\left|\overline{B_{\ell} S_{\ell}}\right|=(1-\lambda) \frac{\Delta x}{2}, \quad\left|\overline{R_{\ell}^{\prime} B_{\ell}^{\prime}}\right|=\lambda^{\prime} \frac{\Delta x}{2}, \quad\left|\overline{B_{\ell}^{\prime} S_{\ell}^{\prime}}\right|=\left(1-\lambda^{\prime}\right) \frac{\Delta x}{2}
$$

Here $\lambda$ and $\lambda^{\prime}$ are adjustable parameters with $0 \leq \lambda, \lambda^{\prime} \leq 1$. Obviously $\left|\overline{R_{\ell} S_{\ell}}\right|=$ $\left|\overline{R_{\ell}^{\prime} S_{\ell}^{\prime}}\right|=\Delta x / 2$. Furthermore, because $\left|\overline{A_{\ell} B_{\ell}}\right|=\left|\overline{B_{\ell} C_{\ell}}\right|=\Delta x / 2$, one concludes that, for $\ell=0,1,2, \ldots$, points $R_{\ell}$ and $S_{\ell}$, respectively, coincide with (i) points $A_{\ell}$ and $B_{\ell}$ if $\lambda=1$, and (ii) points $B_{\ell}$ and $C_{\ell}$ if $\lambda=0$. As a result, as long as $0 \leq \lambda \leq 1$, for each $\ell=1,3,5, \ldots$, the interior of $\overline{R_{\ell} S_{\ell}}$ lies entirely within the solution element associated with point $B_{\ell}$. Obviously the above conclusions also hold if points $R_{\ell}$, $S_{\ell}, A_{\ell}, B_{\ell}$ and $C_{\ell}$, and the parameters $\lambda$ are replaced by points $R_{\ell}^{\prime}, S_{\ell}^{\prime}, A_{\ell}^{\prime}, B_{\ell}^{\prime}$ and $C_{\ell}^{\prime}$, and the parameter $\lambda^{\prime}$, respectively.

The third set of NRBCs will be constructed assuming

$$
F\left(\overline{A_{\ell} A_{\ell+2}}\right)-F\left(\overline{B_{\ell} B_{\ell+2}}\right)=F\left(\overline{R_{\ell-1} R_{\ell+1}}\right)-F\left(\overline{S_{\ell-1} S_{\ell+1}}\right), \quad \ell=2,4,6, \ldots
$$

Note that, because (i) the line segments $\overline{A_{\ell} A_{\ell+2}}, \overline{B_{\ell} B_{\ell+2}}, \overline{R_{\ell-1} R_{\ell+1}}$, and $\overline{S_{\ell-1} S_{\ell+1}}$ have the same length (i.e., $\Delta t$ ), and (ii) the horizontal distance (i.e., $\Delta x / 2$ ) that separates the line segment $\overline{A_{\ell} A_{\ell+2}}$ and $\overline{B_{\ell} B_{\ell+2}}$ is identical to that which separates the line segments $\overline{R_{\ell-1} R_{\ell+1}}$ and $\overline{S_{\ell-1} S_{\ell+1}}$, Eq. (4.26) represents a linear flux extrapolation relation. Also because the rectangles $A_{\ell} A_{\ell+2} B_{\ell+2} B_{\ell}$ and $R_{\ell-1} R_{\ell+1} S_{\ell+1} S_{\ell-1}$ are GCEs, we have Eq. (4.9) and

$$
F\left(\overline{R_{\ell-1} R_{\ell+1}}\right)-F\left(\overline{S_{\ell-1} S_{\ell+1}}\right)+F\left(\overline{R_{\ell+1} S_{\ell+1}}\right)-F\left(\overline{R_{\ell-1} S_{\ell-1}}\right)=0
$$

for $\ell=2,4,6, \ldots$ With the aid of Eqs. (4.9) and (4.27), Eq. (4.26) implies that

$$
F\left(\overline{A_{\ell+2} B_{\ell+2}}\right)-F\left(\overline{R_{\ell+1} S_{\ell+1}}\right)=F\left(\overline{A_{\ell} B_{\ell}}\right)-F\left(\overline{R_{\ell-1} S_{\ell-1}}\right), \quad \ell=2,4,6, \ldots
$$

As a result,

$$
F\left(\overline{A_{\ell} B_{\ell}}\right)-F\left(\overline{R_{\ell-1} S_{\ell-1}}\right)=F\left(\overline{A_{2} B_{2}}\right)-F\left(\overline{R_{1} S_{1}}\right), \quad \ell=4,6,8, \ldots
$$

With the aid of Eqs. (2.4), (2.5), (2.9), and (4.25), it can be shown that Eq. (4.29) is equivalent to

$$
\begin{aligned}
& \left(u_{A_{\ell}}-u_{B_{\ell-1}}\right)-\left[\left(u_{x}^{+}\right)_{A_{\ell}}+(2 \lambda-1)\left(u_{x}^{+}\right)_{B_{\ell-1}}\right]=\left(u_{A_{2}}-u_{B_{1}}\right) \\
& -\left[\left(u_{x}^{+}\right)_{A_{2}}+(2 \lambda-1)\left(u_{x}^{+}\right)_{B_{1}}\right], \quad \ell=4,6,8, \ldots
\end{aligned}
$$

Similarily, the counterpart to Eq. (4.30) for the left boundary is

$$
\begin{aligned}
& \left(u_{A_{\ell}^{\prime}}-u_{B_{\ell-1}^{\prime}}\right)+\left[\left(u_{x}^{+}\right)_{A_{\ell}^{\prime}}+\left(2 \lambda^{\prime}-1\right)\left(u_{x}^{+}\right)_{B_{\ell-1}^{\prime}}\right]=\left(u_{A_{2}^{\prime}}-u_{B_{1}^{\prime}}\right) \\
& +\left[\left(u_{x}^{+}\right)_{A_{2}^{\prime}}+\left(2 \lambda^{\prime}-1\right)\left(u_{x}^{+}\right)_{B_{1}^{\prime}}\right], \quad \ell=4,6,8, \ldots
\end{aligned}
$$

To proceed, note that: (i) Eqs. (4.3) and (4.4) coupled with the assumption $j_{b} \gg 1$ imply that, for Solution I,

$$
u_{j}^{0}=U \quad \text { and } \quad\left(u_{x}^{+}\right)_{j}^{0}=0, \quad j=j_{b}, j_{b} \pm 1
$$


and (ii) each $a^{\prime}$ scheme has the property that, for any $(j, n) \in \Omega, u_{j}^{n}=u_{j \pm 1 / 2}^{n-1 / 2}$ and $\left(u_{x}^{+}\right)_{j}^{n}=0$ if $u_{j-1 / 2}^{n-1 / 2}=u_{j+1 / 2}^{n-1 / 2}$ and $\left(u_{x}^{+}\right)_{j \pm 1 / 2}^{n-1 / 2}=0$. As a result, for Solution I,

$$
u_{j_{b} \pm 1 / 2}^{1 / 2}=U \text { and } \quad\left(u_{x}^{+}\right)_{j_{b} \pm 1 / 2}^{1 / 2}=0
$$

and

$$
u_{j_{b}}^{1}=U \quad \text { and } \quad\left(u_{x}^{+}\right)_{j_{b}}^{1}=0
$$

Because, in Figs. 8 and 9, the mesh points $\left(j_{b}-1 / 2,1 / 2\right)$ and $\left(j_{b}, 1\right)$ are denoted by $B_{1}$ and $A_{2}$, respectively, Eqs. (4.33) and (4.34) imply that, for Solution I,

$$
u_{A_{2}}=u_{B_{1}}=U \text { and }\left(u_{x}^{+}\right)_{A_{2}}=\left(u_{x}^{+}\right)_{B_{1}}=0
$$

Similarly, it can be shown that, for Solution I,

$$
u_{A_{2}^{\prime}}=u_{B_{1}^{\prime}}=V \quad \text { and } \quad\left(u_{x}^{+}\right)_{A_{2}^{\prime}}=\left(u_{x}^{+}\right)_{B_{1}^{\prime}}=0
$$

It will be assumed that Eqs. (4.35) and (4.36) are also valid for Problem II. As a result, the expressions on the right sides of Eqs. (4.30) and (4.31) vanish.

Let the parameter $\lambda$ and the marching variables $u_{B_{\ell-1}}$ and $\left(u_{x}^{+}\right)_{B_{\ell-1}}$ (which are associated with a lower time level) in Eq. (4.30) be given. Then, for each $\ell=4,6,8, \ldots$, there are only two unknowns, i.e., the boundary data $u_{A_{\ell}}$ and $\left(u_{x}^{+}\right)_{A_{\ell}}$ in Eq. (4.30). As a result, after imposing Eq. (4.30), there is still one degree of freedom left in choosing the two unknowns. To obtain the simplest NRBCs, the degree of freedom is removed by further assuming that the sum of the zero-order terms and that of the first-order terms on the left side of Eq. (4.30), respectuively, are equal to zero. Thus one has

$$
u_{A_{\ell}}=u_{B_{\ell-1}} \quad \text { and } \quad\left(u_{x}^{+}\right)_{A_{\ell}}=(1-2 \lambda)\left(u_{x}^{+}\right)_{B_{\ell-1}} \quad \ell=2,4,6, \ldots
$$

Similarly, the counterpart of Eq. (4.37) for the left boundary is

$$
u_{A_{\ell}^{\prime}}=u_{B_{\ell-1}^{\prime}} \text { and }\left(u_{x}^{+}\right)_{A_{\ell}^{\prime}}=\left(1-2 \lambda^{\prime}\right)\left(u_{x}^{+}\right)_{B_{\ell-1}^{\prime}} \quad \ell=2,4,6, \ldots
$$

Note that: (i) obviously Eq. (4.24) must also be observed here; and (ii) hereafter Eqs. (4.37) and (4.38) shall be designated as the third set of NRBCs.

4.4. The Fourth Set of NRBCs. Each of the three sets of NRBCs introduced above represents only the simplest among many possible combinations of boundary conditions that are consistent with the associated flux extrapolation conditions. As an example, instead of using the simplest approach described in Sec. 4.2, nonuniqueness involving Eqs. (4.20) and (4.21) can also be removed by assuming that, for $\ell=2,4,6 \ldots$,

$$
u_{A_{\ell}}=u_{C_{\ell}}+\Delta x\left(u_{x}\right)_{C_{\ell}} \quad \text { and } \quad u_{A_{\ell}^{\prime}}=u_{C_{\ell}^{\prime}}-\Delta x\left(u_{x}\right)_{C_{\ell}^{\prime}}, \quad \ell=2,4,6, \ldots
$$

i.e., $u_{A_{\ell}}$ and $u_{A_{\ell}^{\prime}}$ are related to $u_{C_{\ell}}$ and $u_{C_{\ell}^{\prime}}$, respectively, by the first-order Taylor's expansion. Combining Eqs. (4.20), (4.21), and (4.39), and the fact that the expressions on the right sides of Eqs. (4.20) and (4.21) vanish, it can be shown that

$$
\left(u_{x}^{+}\right)_{A_{\ell}}=(5-2 \lambda)\left(u_{x}^{+}\right)_{C_{\ell}} \quad \text { and } \quad\left(u_{x}^{+}\right)_{A_{\ell}^{\prime}}=\left(5-2 \lambda^{\prime}\right)\left(u_{x}^{+}\right)_{C_{\ell}^{\prime}}, \quad \ell=2,4,6, \ldots
$$

Eqs. (4.39) and (4.40) shall be designated as the fourth set of NRBCs. Note that, because $1 \geq \lambda, \lambda^{\prime} \geq 0$, one has

$$
5 \geq(5-2 \lambda),\left(5-2 \lambda^{\prime}\right) \geq 3
$$


4.5. Remarks and Extensions. This section is concluded with the following comments:

(a) To simplify discussion, it is assumed in Eqs. (4.3)-(4.6) that the initial source of disturbance is a single discontinuity which is at a reasonable distance away from the mesh points $\left(0, \pm j_{b}\right)$. However, according to the logic of the current development, the NRBCs derived here are still valid even if the discontinuity is replaced by a source region of finite extent as long as this source region is kept at a reasonable distance away from any nonreflecting boundary.

(b) With the aid of Eqs. (2.11)-(2.15) and the related remarks, the development presented in this section can be extended to the 1D Euler case in a straightforward manner. Specifically, in the Euler development, (i) Eq. (2.1) is replaced by Eq. (2.11); (ii) Eqs. (4.1) and (4.3)-(4.6), respectively, are replaced by their Euler versions that, respectively, are identical to these equations except that $u, u_{j}^{0},\left(u_{x}^{+}\right)_{j}^{0}, U$, and $V$ respectively, are replaced by $u_{m}$, $\left(u_{m}\right)_{j}^{0},\left(u_{m x}^{+}\right)_{j}^{0}, U_{m}$, and $V_{m}$, where $m=1,2,3$, and $U_{m}$ and $V_{m}$ are two different constants for each $m$; (iii) the exact solution given in Eq. (4.2) is replaced by its Euler version, i.e., the Riemann solution [41, p. 181]; and (iv) the condition $0 \leq \lambda, \lambda^{\prime} \leq 1$ is again assumed. Using the new definitions given in the above item (ii), one can proceed to define the Euler versions of Problems I and II, and Solutions I and II. Obviously, (i) by replacing the symbols $u$ and $u_{x}^{+}$with $\left(u_{m}\right)$ and $\left(u_{m x}^{+}\right)$, respectively, the NRBCs and $\left(u_{m x}^{+}\right)$, respectively, the NRBCs Eqs. (4.12)-(4.15), (4.20)-(4.23), (4.30), (4.31), and (4.37)-(4.40) will become their Euler versions, respectively. Note that hereafter the Euler version of an equation such as Eq. (4.3) will be denoted by Eq. (E-4.3).

(c) Consider the 1D Euler case. Because $m=1,2,3$, each of Eqs. (E-4.12), (E-4.13), (E-4.20), (E-4.21), (E-4.30), and (E-4.31) represents a set of three conditions involving six unknowns. Thus, after imposing any one of these equations at a boundary mesh point $(j, n)$, there are still three degrees left in specifying $\left(u_{m}\right)_{j}^{n}$ and $\left(u_{m x}^{+}\right)_{j}^{n}$. The several approaches used here to eliminate these degrees of freedom represent only a small subset of all possible options. It is conceivable that, by using other options, one may be able to construct a set of NRBCs that also meet some extra given conditions at the boundary.

\section{Numerical Results}

In this section, the Euler versions of the four sets of NRBCs developed in Sec. 4 will be validated numerically. Because only the Euler versions are considered here, in this section a phrase such as "the Euler version of Problem I" will be abbreviated simply as "Problem I".

The 1D test problem to be specified shortly is an extended Sod's shock tube problem. It is the original Sod's problem [42] with the additional complication of imposing NRBCs at the two open ends of the shock tube. Note that the flow under consideration contains a shock wave and a contact discontinuity, and, relative to the computational frame, is subsonic throughout (the values of Mach number range from 0 to 0.93). It is well known that implementing NRBCs for a subsonic flow is much more difficult than doing the same for a supersonic flow. This difficulty is further exacerbated by the fact that most established NRBCs are derived assuming a continuous flow-which is not valid for the current case. In this section, each of 
the four sets of NRBCs developed in Section 4 will be validated by comparing the resulting numerical solutions against the exact analytical solutions for a period of time that ends only after the shock wave and contact discontinuity have exited the computational domain.

To proceed, let the meshes used by Problems I and II, respectively, be those depicted in Figs. 6 and 7 with $\Delta t=0.004, \Delta x=0.01$ and $j_{0}=50$. Thus, for Problem II, $j_{b}=50 \frac{1}{2}$ and $-0.505 \leq x \leq 0.505$. In addition, it is assumed that: (i) the specific heat ratio $\gamma=1.4$; (ii) both Solutions I and II are obtained using the 1D Euler $a-\alpha$ scheme with $\alpha=1$; and (iii) the initial conditions at $t=0$ for Problem I (Problem II) are Eqs. (E-4.3) and (E-4.4) (Eqs. (E-4.5) and (E-4.6)) with $\left(U_{1}, U_{2}, U_{3}\right)=(0.125,0,0.25)$ and $\left(V_{1}, V_{2}, V_{3}\right)=(1,0,2.5)$. For both Solutions I and II, the above assumptions imply a Courant number $C F L \simeq 0.88$ where $C F L$ is defined to be the maximal numerical value of of $(|v|+c) \Delta t / \Delta x$ with $v$ and $c$ being the local velocity and sonic speed, respectively. Note that stability of the 1D Euler $a-\alpha$ scheme generally requires $C F L<1$ and $\alpha \geq 0$.

Moreover, by using (i) Fig. 6; (ii) Eqs. (E-4.3) and (E-4.4); and (iii) a property of the 1D Euler $a-\alpha$ scheme, i.e., for any $(j, n) \in \Omega,\left(u_{m}\right)_{j}^{n}=\left(u_{m}\right)_{j \pm 1 / 2}^{n-1 / 2}$ and $\left(u_{m x}^{+}\right)_{j}^{n}=0$ if $\left(u_{m}\right)_{j-1 / 2}^{n-1 / 2}=\left(u_{m}\right)_{j+1 / 2}^{n-1 / 2}$ and $\left(u_{m x}^{+}\right)_{j \pm 1 / 2}^{n-1 / 2}=0$, it can be shown that, for Solution I,

and

$$
\left(u_{m}\right)_{j}^{n}=U_{m} \quad \text { and } \quad\left(u_{m x}^{+}\right)_{j}^{n}=0 \quad \text { if } \quad j>n
$$

In other words, the impact of the initial discontinuity is felt only in the mesh region $|j|<n$ (note: $|j| \neq n$ if $(j, n) \in \Omega$ ). Because $j_{b}=j_{0}+1 / 2$, Eqs. (5.1) and (5.2) imply that

$$
\left(u_{m}\right)_{j_{b}}^{n}=U_{m} \quad \text { and } \quad\left(u_{m x}^{+}\right)_{j_{b}}^{n}=0, \quad n=0,1,2, \ldots, j_{0}
$$

and

$$
\left(u_{m}\right)_{-j_{b}}^{n}=V_{m} \quad \text { and } \quad\left(u_{m x}^{+}\right)_{-j_{b}}^{n}=0, \quad n=0,1,2, \ldots, j_{0}
$$

respectively. Furthermore, because (i) $n=\ell / 2$, (ii) $\left(u_{m}\right)_{A_{0}}=\left(u_{m}\right)_{j_{b}}^{0}=U_{m}$ and $\left(u_{m x}^{+}\right)_{A_{0}}=\left(u_{m x}^{+}\right)_{j_{b}}^{0}=0$, and (iii) $\left(u_{m}\right)_{A_{0}^{\prime}}=\left(u_{m}\right)_{-j_{b}}^{0}=V_{m}$ and $\left(u_{m x}^{+}\right)_{A_{0}^{\prime}}=$ $\left(u_{m x}^{+}\right)_{-j_{b}}^{0}=0$, a comparison of Eqs. (E-4.14) and (E-4.15) with Eqs. (5.3) and (5.4) reveals that, for $n=0,1,2, \ldots, j_{0}$, the boundary values at the mesh lines $j= \pm j_{b}$ specified using the first set of NRBCs are identical to those specified using the solution values of Solution I. According to a discussion given in Sec. 4.1, this implies that Solution II coincides with Solution I at all mesh points $(j, n)$ with $|j| \leq j_{b}$ and $0 \leq n \leq j_{0}$ if the boundary values of Problem II are specified using the first set of NRBCs. Note that, given any pair of the values of $\lambda$ and $\lambda^{\prime}$, one can also show that: (i) Solution II coincides with Solution I at all mesh points $(j, n)$ with $|j| \leq j_{b}$ and $0 \leq n \leq j_{0}$ if the third set of NRBCs is used; and (ii) excluding the two boundary mesh points with $j= \pm j_{b}$ and $n=j_{0}$, Solution II coincides with Solution I at all mesh points $(j, n)$ with $|j| \leq j_{b}$ and $0 \leq n \leq j_{0}$ if the second or the fourth set of NRBCs is used.

Because $j_{0}=50$ and $\Delta t=0.004, t=n \Delta t=0.2$ when $n=j_{0}$. As a result, the above discussions imply that, within their common spatial domain, Solution II should be identical to Solution I in the time interval $0 \leq t \leq 0.2$ if the first or 
the third set of NRBCs is used. Also, except for the two boundary mesh points mentioned above, the above conclusion also applies if the second or the fourth set of NRBCs is used. Note that, in reality, the differences between Solutions I and II are completely negligible at the two exceptional boundary mesh points.

At $t=0.2$, Solution II generated using the first set of NRBCs is shown in Fig. 10(a). In this figure, the numerical values (marked by triangles) of the density, the velocity and the pressure are compared with the exact solutions (marked by solid lines). As expected from the discussions given in the last paragraph, at $t=$ 0.2 , Solution II generated using any one of the other three sets of NRBCs is also represented by the same results shown in Fig. 10(a). Note that, at $t=0.2$, the waves and shocks generated in the interior have not yet reached the boundaries. Also it is seen that the agreement between the numerical results and the exact solutions is excellent. In particular, the shock discontinuity is resolved almost within one mesh interval, and the contact discontinuity is resolved in four mesh intervals. Also, there are only slight numerical overshoots and/or oscillations near these discontinuities.

At $t=0.4$, Solution II generated using the first set of NRBCs is shown in Fig. 10(b). It is seen that, by this time, the shock wave has passed cleanly through the right boundary. There is good agreement between the numerical solutions and the exact solutions everywhere in the interior except for a slight disagreement in the vicinity of the right boundary. Note that the right boundary values, which do not vary with time and are no longer identical to the values of Solution $I$ there at $t=0.4$, are discontinuous with respect to the neighboring interior values. The numerical results at $t=0.6$ are shown in Fig. 10(c). As seen from the density profile, by this time, the contact discontinuity has also passed through the right boundary. Agreement between the numerical solutions and the analytical solutions continues to be good in the interior. However, both left and right boundary values are now discontinuous with respect to the neighboring interior values.

At $t=0.2,0.4,0.6$, solution II generated using the third set of NRBCs with $\lambda=$ $\lambda^{\prime}=0$ are shown in Figs. 10(a), 11(a) and 11(b), respectively. The agreement with the exact solution is excellent everywhere. In particular, unlike the case associated with the cruder first set of NRBCs, agreement between the numerical and exact solutions at $t=0.4$ and $t=0.6$ are quite good for the current case even at the right boundary. Note that the use of other values of $\lambda$ and $\lambda^{\prime}$ in the range $0 \leq \lambda, \lambda^{\prime} \leq 1$ yields almost identical numerical results.

To give the reader a concrete idea about the simplicity of the CE/SE method in general and the current NRBCs in particular, the self-contained Fortran program which is used to generate the results depicted in Figs. 10(a), 11(a) and 11(b) is listed in Appendix. In this program, the input and output include: (i) $\mathrm{nx}=2 j_{b}$ $=$ the number of total mesh intervals at the 0th time level, (ii) it $=$ the number of total marching steps (each marching step advances a time period of $\Delta t / 2$ ), (iii) dt $=\Delta t$, (iv) $\mathrm{dx}=\Delta x,(\mathrm{v})(\mathrm{u} 1, \mathrm{u} 2, \mathrm{u} 3)=\left(U_{1}, U_{2}, U_{3}\right),(\mathrm{vi})(\mathrm{v} 1, \mathrm{v} 2, \mathrm{v} 3)=\left(V_{1}, V_{2}, V_{3}\right)$, (vii) $\mathrm{cr}=1-2 \lambda$, (viii) $\mathrm{cl}=1-2 \lambda^{\prime}$, (ix) ia $=\alpha$, (x) rho = mass density, (xi) $\mathrm{v}=$ fluid velocity, (xii) $\mathrm{p}=$ static pressure, and (xiii) $\mathrm{M}=$ Mach number. Note that $(1-2 \lambda)$ and $\left(1-2 \lambda^{\prime}\right)$ are the factors that appear in Eqs. (4.37) and (4.38), respectively. Thus, according to Eq. (4.24), $1 \geq \mathrm{cr}, \mathrm{cl} \geq-1$.

Solution II also has been generated using the second and the fourth sets of NRBCs with different values of $\lambda$ and $\lambda^{\prime}$. At $t=0.2,0.4,0.6$, the numerical results thus obtained are virtually indistingushible (at least visually) from those shown in Fig. 10(a), 11(a) and 11(b) for all $\lambda$ and $\lambda^{\prime}$ in the range $0 \leq \lambda, \lambda^{\prime} \leq 1$. 
Note that the second, the third and the fourth sets of NRBCs were derived assuming $0 \leq \lambda, \lambda^{\prime} \leq 1$. As a result, breakdown of these NRBCs is expected if the values of $\lambda$ and $\lambda^{\prime}$ used fall outside of the range $0 \leq \lambda, \lambda^{\prime} \leq 1$. As an example, consider the fourth set of NRBCs with $\lambda=\lambda^{\prime}=2$. For this special case, Eqs. (E4.39) and (E-4.40) can be rearranged as

$$
\left(u_{m}\right)_{A_{\ell}}=\left(u_{m}\right)_{C_{\ell}}+\Delta x\left(u_{m x}\right)_{C_{\ell}} \quad \text { and } \quad\left(u_{m x}^{+}\right)_{A_{\ell}}=\left(u_{m x}^{+}\right)_{C_{\ell}}
$$

and

$$
\left(u_{m}\right)_{A_{\ell}^{\prime}}=\left(u_{m}\right)_{C_{\ell}^{\prime}}-\Delta x\left(u_{m x}\right)_{C_{\ell}^{\prime}} \text { and }\left(u_{m x}^{+}\right)_{A_{\ell}^{\prime}}=\left(u_{m x}^{+}\right)_{C_{\ell}^{\prime}}
$$

where $m=1,2,3$ and $\ell=2,4,6, \ldots$ Because (i) $\left(u_{m}\right)_{A_{\ell}}$ and $\left(u_{m}\right)_{A_{\ell}^{\prime}}$ are related to $\left(u_{m}\right)_{C_{\ell}}$ and $\left(u_{m}\right)_{C_{\ell}^{\prime}}$, respectively, by the first-order Taylor's expansion; and (ii) $\left(u_{m x}^{+}\right)_{A_{\ell}}$ and $\left(u_{m x}^{+}\right)_{A_{\ell}^{\prime}}$ simply assume the values of the corresponding variables at the neighboring mesh points $C_{\ell}$ and $C_{\ell}^{\prime}$, respectively, these boundary conditions certainly look "reasonable". As explained earlier, at $t=0.2$, Solution II generated using Eqs. (5.5) and (5.6) is again represented by those shown in Fig. 10(a). However, at $t=0.4$ (see Fig. 12), Solution II generated using these "reasonable" boundary conditions has become highly reflecting at the right boundary.

\section{Conclusions and Discussions}

In this paper, we first review the CE/SE schemes for the $1 \mathrm{D}$ advection equation Eq. (2.1) and those for the 1D Euler equations Eq. (2.11). The concept of a generalized conservation element was then developed, paving the way for the development of flux-based NRBCs.

Four sets of simple NRBCs for the advection equation were developed on the basis of extrapolation of fluxes at the boundaries. No characteristics-based techniques were used in the development. The Euler versions of these NRBCs were developed in a similar manner and tested with the 1D Euler $a-\alpha$ scheme. The test problem used is an extension of the original Sod's shock-tube problem in which extra NRBCs are imposed at the two open ends of the shock tube.

The first set of Euler NRBCs is the simplest and represents a set of steadystate boundary conditions. It was truly remarkable that this set of NRBCs yielded numerical solutions that were in good agreement with the exact weak solution in the interior of the computational domain, even after the prescribed steady-state boundary values had deviated completely from those associated with the exact solution.

It was also shown that the more advanced second, third and fourth sets of Euler NRBCs yielded numerical solutions that were in even better agreement with the exact weak solution -if the values of the adjustable parameters $\lambda$ and $\lambda^{\prime}$ used in these NRBCs fell within the allowable range $0 \leq \lambda, \lambda^{\prime} \leq 1$. However, as shown by a numerical example, strong spurious reflections from a boundary may occur if the values of $\lambda$ and $\lambda^{\prime}$ chosen fall outside the allowable range.

Finally note that it has been shown numerically $[19,21-28]$ that the multidimensional extensions of the current Euler NRBCs are also equally simple, effective and robust. In particular, the reader is referred to [19] for a rigorous discussion of the investigation of several classes of flow problems using a 2D CE/SE Euler solver [15] in conjunction with a 2D extension of the present 1D Euler NRBCs. The classes of problems considered include (i) acoustic pulse, entropy wave, and 
vorticity wave propagation, (ii) free shear-layer instability, and (iii) multiple interactions of a strong vortex and strong oblique shocks. The major conclusions of this investigation are:

(a) The (second-order) 2D CE/SE solver is efficient and yields high resolution, low dispersion results similar to those of higher-order conventional schemes [4-6].

(b) The novel 2D CE/SE NRBCs used are easy to implement and remove the need for (or mimimize the size of) a buffer zone.

(c) The 2D CE/SE solver is capable of handling both continuous and discontinuous flows and, thus, provides a unique numerical tool for flow situations where sound waves and shocks and their interactions are important, such as the jet screech noise problem.

\section{Appendix: A Solver for An Extended Sod's Shock-Tube Problem}

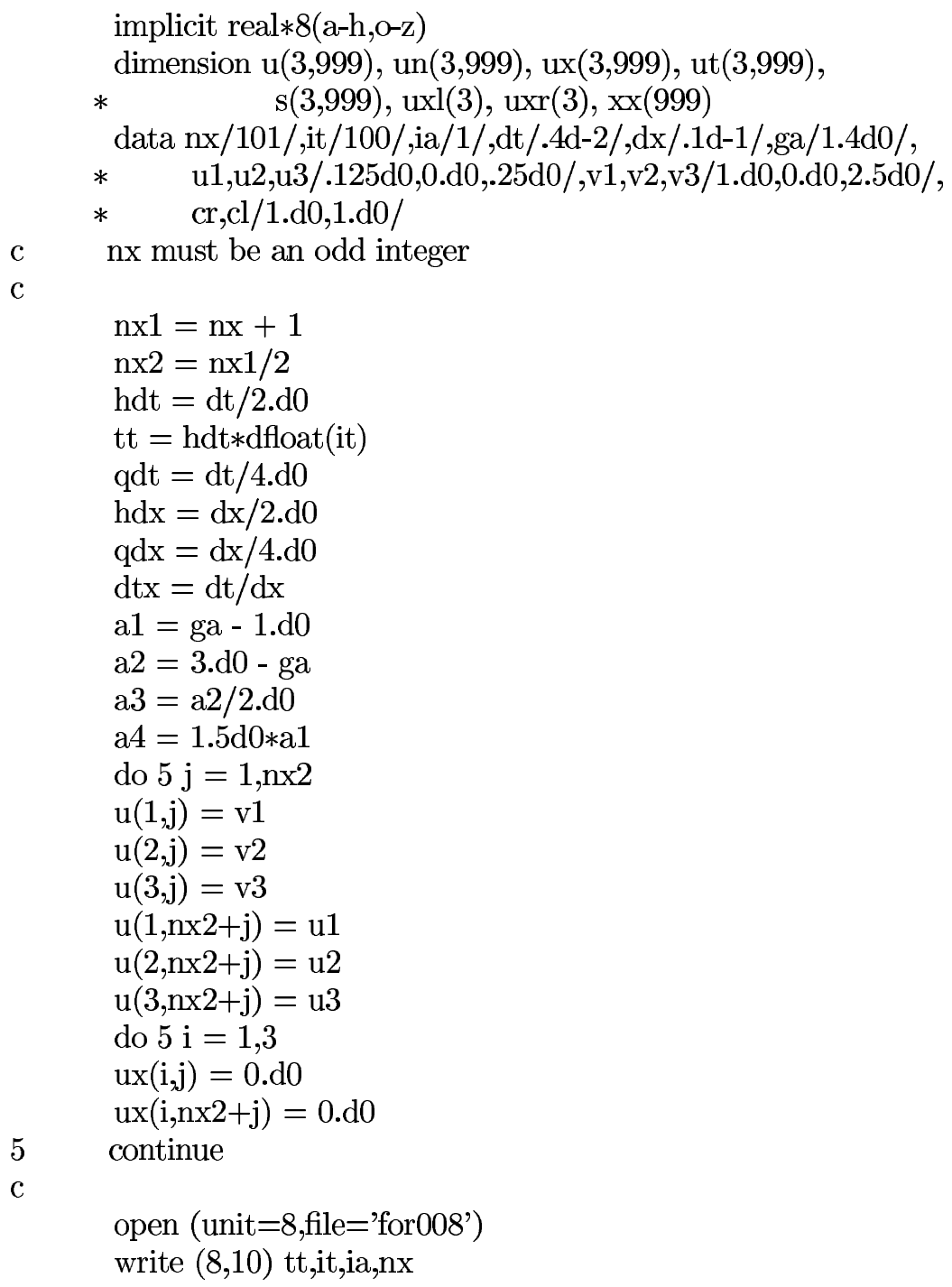


write $(8,20) \mathrm{dt}, \mathrm{dx}, \mathrm{ga}$

write $(8,30) \mathrm{u} 1, \mathrm{u} 2, \mathrm{u} 3, \mathrm{cr}$

write $(8,40)$ v1,v2,v3,cl

$\mathrm{c}$

do $400 \mathrm{i}=1$,it

$\mathrm{m}=\mathrm{nx}+\mathrm{i}-(\mathrm{i} / 2) * 2$

do $100 \mathrm{j}=1, \mathrm{~m}$

$\mathrm{w} 2=\mathrm{u}(2, \mathrm{j}) / \mathrm{u}(1, \mathrm{j})$

$\mathrm{w} 3=\mathrm{u}(3, \mathrm{j}) / \mathrm{u}(1, \mathrm{j})$

$\mathrm{f} 21=-\mathrm{a} 3 * \mathrm{w} 2 * * 2$

$\mathrm{f} 22=\mathrm{a} 2 * \mathrm{w} 2$

$\mathrm{f} 31=\mathrm{a} 1 * \mathrm{w} 2 * * 3-\mathrm{ga} * \mathrm{w} 2 * \mathrm{w} 3$

$\mathrm{f} 32=\mathrm{ga} * \mathrm{w} 3-\mathrm{a} 4 * \mathrm{w} 2 * * 2$

$\mathrm{f} 33=\mathrm{ga} * \mathrm{w} 2$

$\mathrm{ut}(1, \mathrm{j})=-\mathrm{ux}(2, \mathrm{j})$

$\mathrm{ut}(2, \mathrm{j})=-(\mathrm{f} 21 * \mathrm{ux}(1, \mathrm{j})+\mathrm{f} 22 * \mathrm{ux}(2, \mathrm{j})+\mathrm{a} 1 * \mathrm{ux}(3, \mathrm{j}))$

$\mathrm{ut}(3, \mathrm{j})=-(\mathrm{f} 31 * \mathrm{ux}(1, \mathrm{j})+\mathrm{f} 32 * \mathrm{ux}(2, \mathrm{j})+\mathrm{f} 33 * \mathrm{ux}(3, \mathrm{j}))$

$\mathrm{s}(1, \mathrm{j})=\mathrm{qdx} * \mathrm{ux}(1, \mathrm{j})+\mathrm{dtx} *(\mathrm{u}(2, \mathrm{j})+\mathrm{qdt} * \mathrm{ut}(2, \mathrm{j}))$

$\mathrm{s}(2, \mathrm{j})=\mathrm{qdx} * \mathrm{ux}(2, \mathrm{j})+\mathrm{dtx} *(\mathrm{f} 21 *(\mathrm{u}(1, \mathrm{j})+\mathrm{qdt} * \mathrm{ut}(1, \mathrm{j}))+$

* $\quad \mathrm{f} 22 *(\mathrm{u}(2, \mathrm{j})+\mathrm{qdt} * \mathrm{ut}(2, \mathrm{j}))+\mathrm{a} 1 *(\mathrm{u}(3, \mathrm{j})+\mathrm{qdt} * \mathrm{ut}(3, \mathrm{j})))$

$\mathrm{s}(3, \mathrm{j})=\mathrm{qdx} * \mathrm{ux}(3, \mathrm{j})+\mathrm{dtx} *(\mathrm{f} 31 *(\mathrm{u}(1, \mathrm{j})+\mathrm{qdt} * \mathrm{ut}(1, \mathrm{j}))+$

100

* $\quad \mathrm{f} 32 *(\mathrm{u}(2, \mathrm{j})+\mathrm{qdt} * \mathrm{ut}(2, \mathrm{j}))+\mathrm{f} 33 *(\mathrm{u}(3, \mathrm{j})+\mathrm{qdt} * \mathrm{ut}(3, \mathrm{j})))$

continue

if (i.ne. $(\mathrm{i} / 2) * 2)$ goto 150

do $120 \mathrm{k}=1,3$

$\mathrm{ux}(\mathrm{k}, 1)=\mathrm{cl} * \mathrm{ux}(\mathrm{k}, 1)$

$\mathrm{ux}(\mathrm{k}, \mathrm{nx} 1)=\mathrm{cr} * \mathrm{ux}(\mathrm{k}, \mathrm{nx})$

$\mathrm{un}(\mathrm{k}, 1)=\mathrm{u}(\mathrm{k}, 1)$

$\mathrm{un}(\mathrm{k}, \mathrm{nx} 1)=\mathrm{u}(\mathrm{k}, \mathrm{nx})$

120

continue

$150 \quad \mathrm{j} 1=1-\mathrm{i}+(\mathrm{i} / 2) * 2$

$\mathrm{mm}=\mathrm{m}-1$

do $200 \mathrm{j}=1, \mathrm{~mm}$

do $200 \mathrm{k}=1,3$

$\mathrm{un}(\mathrm{k}, \mathrm{j}+\mathrm{j} 1)=0.5 \mathrm{~d} 0 *(\mathrm{u}(\mathrm{k}, \mathrm{j})+\mathrm{u}(\mathrm{k}, \mathrm{j}+1)+\mathrm{s}(\mathrm{k}, \mathrm{j})-\mathrm{s}(\mathrm{k}, \mathrm{j}+1))$

$\mathrm{uxl}(\mathrm{k})=(\mathrm{un}(\mathrm{k}, \mathrm{j}+\mathrm{j} 1)-\mathrm{u}(\mathrm{k}, \mathrm{j})-\mathrm{hdt} * \mathrm{ut}(\mathrm{k}, \mathrm{j})) / \mathrm{hdx}$

$\mathrm{uxr}(\mathrm{k})=(\mathrm{u}(\mathrm{k}, \mathrm{j}+1)+\mathrm{hdt} * \mathrm{ut}(\mathrm{k}, \mathrm{j}+1)-\mathrm{un}(\mathrm{k}, \mathrm{j}+\mathrm{j} 1)) / \mathrm{hdx}$

$\mathrm{ux}(\mathrm{k}, \mathrm{j}+\mathrm{j} 1)=(\mathrm{uxl}(\mathrm{k}) *(\operatorname{dabs}(\mathrm{uxr}(\mathrm{k}))) * * \mathrm{ia}+\mathrm{uxr}(\mathrm{k}) *(\operatorname{dabs}(\mathrm{uxl}(\mathrm{k})))$

200

* $* * \mathrm{ia}) /((\operatorname{dabs}(\mathrm{uxl}(\mathrm{k}))) * * \mathrm{ia}+(\operatorname{dabs}(\operatorname{uxr}(\mathrm{k}))) * * \mathrm{ia}+1 . \mathrm{d}-60)$

continue

$\mathrm{m}=\mathrm{nx} 1-\mathrm{i}+(\mathrm{i} / 2) * 2$

do $300 \mathrm{j}=1, \mathrm{~m}$

do $300 \mathrm{k}=1,3$

$\mathrm{u}(\mathrm{k}, \mathrm{j})=\mathrm{un}(\mathrm{k}, \mathrm{j})$

300 continue

400 continue

c

$\mathrm{m}=\mathrm{nx} 1-\mathrm{it}+(\mathrm{it} / 2) * 2$

$\mathrm{mm}=\mathrm{m}-1$ 


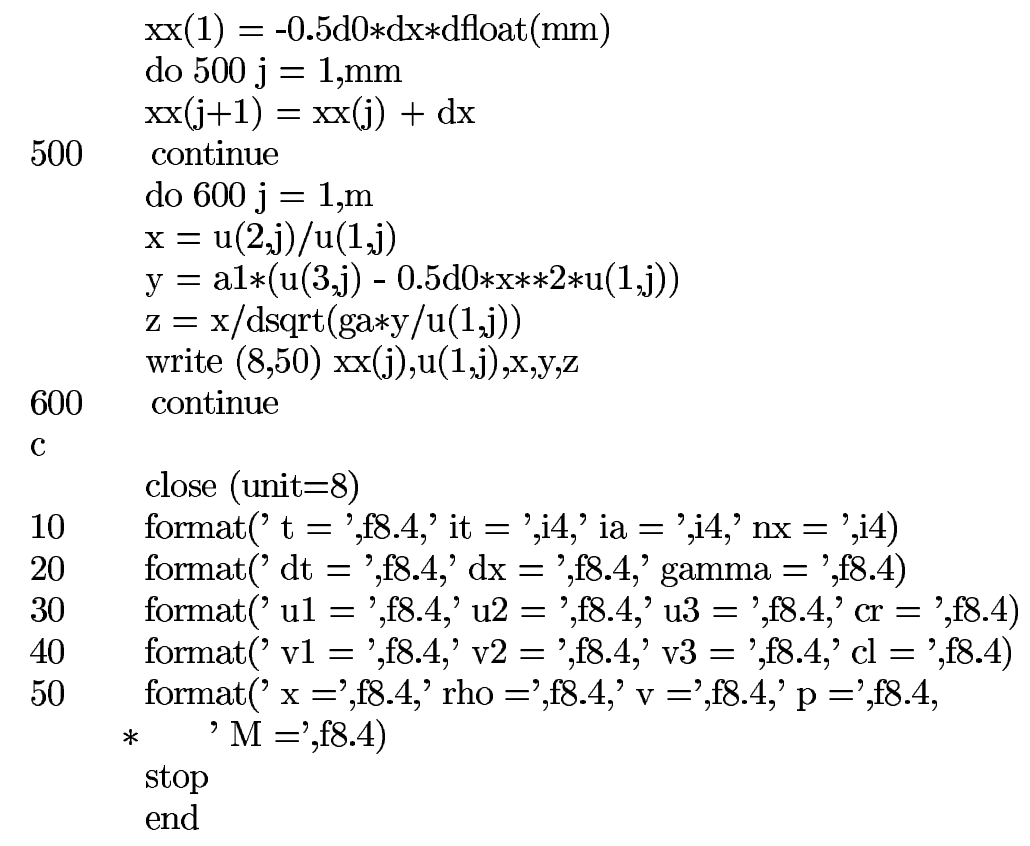

\section{References}

[1] B. Engquist and A. Majda, Absorbing Boundary Conditions for the Numerical Simulation of Waves, Mathematics of Computation, 31(139), 629 (1977).

[2] K.W. Thompson, Time Dependent Boundary Conditions for Hyperbolic Systems, Part I, J. Comput. Phys., 68, 1 (1987).

[3] K.W. Thompson, Time Dependent Boundary Conditions for Hyperbolic Systems, Part II, $J$. Comput. Phys., 89, 439 (1990).

[4] C.K.W. Tam and J.C. Webb, Dispersion-Relation-Preserving Finite Difference Scheme for Computational Acoustics, J. Comput. Phys., 107, 262 (1993).

[5] S.T. Yu, L.S. Hultgren, and N.S. Liu, Direct Calculations of Waves in Fluid Flows Using High Order Compact Difference Scheme, AIAA J., 32(9), 1766 (1994).

[6] H.L. Atkins, Application of Essentially Non-Oscillatory Methods to Aeroacoustics Problems, ICASE / LaRC Workshop on Benchmark Problems in Computational Aeroacoustics, NASA CP 3300 (1994).

[7] F.Q. Hu, On Absorbing Boundary Conditions for Linearlized Euler Equations by A Perfectly Matched Layer, J. Comput. Phys., 129, 201 (1996).

[8] T. Colonius, S.K. Lele, and P. Moin, Sound Generation in a Mixing Layer, Journal of Fluid Mechanics, 330, 375 (1997).

[9] S.C. Chang and W.M. To, A New Numerical Framework for Solving Conservation LawsThe Method of Space-Time Conservation Element and Solution Element, NASA TM 104495, August 1991.

[10] S.C. Chang and W.M. To, A brief description of a new numerical framework for solving conservation laws-The method of space-time conservation element and solution element, in Proceedings of the Thirteenth International Conference on Numerical Methods in Fluid Dynamics, Rome, Italy, 1992, edited by M. Napolitano and F. Sabetta, Lecture Notes in Physics 414, (Springer-Verlag, New York/Berlin, 1992), p. 396.

[11] S.C. Chang, The method of space-time conservation element and solution Element-A new approach for solving the Navier-Stokes and Euler equations, J. Comput. Phys., 119, 295 (1995).

[12] S.C. Chang, S.T. Yu, A. Himansu, X.Y. Wang, C.Y. Chow, and C.Y. Loh, The method of space-time conservation element and solution element-A new paradigm for numerical solution of conservation laws, in Computational Fluid Dynamics Review 1998 edited by M.M. Hafez and K. Oshima (World Scientific, Singapore), Vol. 1, p. 206. 
[13] T. Molls and F. Molls, Space-Time Conservation Method Applied to Saint Venant Equations, J. of Hydraulic Engr., 124(5), 501 (1998).

[14] C. Zoppou and S. Roberts, Space-Time Conservation Method Applied to Saint Venant Equations: A Discussion, J. of Hydraulic Engr., 125(8), 891 (1999).

[15] S.C. Chang, X.Y. Wang, and C.Y. Chow, The space-time conservation element and solution element method: A new high-resolution and genuinely multidimensional paradigm for solving conservation laws, J. Comput. Phys., 156, 89 (1999).

[16] X.Y. Wang, and S.C. Chang, A 2D non-splitting unstructured triangular mesh Euler solver based on the space-time conservation element and solution element method, Computational Fluid Dynamics Journal, 8(2), 309 (1999).

[17] S.C. Chang, X.Y. Wang and W.M. To, Application of the space-time conservation element and solution element method to one-dimensional convection-diffusion problems, J. Comput. Phys., 165, 189 (2000).

[18] J. Qin, S.T. Yu, Z.C. Zhang, and M.C. Lai, Direct Calculations of Cavitating Flows by the Space-Time CE/SE Method, J. Fuels \& Lubricants, SAE Transc., 108(4), 1720 (2000).

[19] C.Y. Loh, L.S. Hultgren and S.C. Chang, Wave computation in compressible flow using the space-time conservation element and solution element method, AIAA J., 39(5), 794 (2001).

[20] Z.C. Zhang, S.T. Yu, and S.C. Chang, A Space-Time Conservation Element and Solution Element Method for Solving the Two- and Three-Dimensional Unsteady Euler Equations Using Quadrilateral and Hexahedral Meshes, J. Comput. Phys., 175, 168 (2002).

[21] K.B.M.Q. Zaman, M.D. Dahl, T.J. Bencic, and C.Y. Loh, Investigation of A 'Transonic Resonance' with Convergent-Divergent Nozzles, J. Fluid Mech., 463, 313 (2002).

[22] C.Y. Loh and K.B.M.Q. Zaman, Numerical Investigation of 'Transonic Resonance' with A Convergent-Divergent Nozzle, AIAA Paper 2002-0077 (accepted for publication in AIAA J.).

[23] C.Y. Loh, L.S. Hultgren and S.C. Chang, Vortex Dynamics Simulation in Aeroacoustics by the Space-Time Conservation Element and Solution Element Method, AIAA Paper 99-0359 (1999)

[24] C.Y. Loh, L.S. Hultgren, S.C. Chang and P.C.E. Jorgenson, Noise Computation of a Supersonic Shock-Containing Axisymmetric Jet by the CE/SE Method, AIAA Paper 2000-0475 (2000).

[25] C.Y. Loh, X.Y. Wang, S.C. Chang, and P.C.E. Jorgenson, Computation of Feedback Aeroacoustic System by the CE/SE Method, in Proceedings of the First International Conference on Computational Fluid Dynamics, Kyoto, Japan, 10-14 July, 2000, edited by N. Satofuka, (Springer-Verlag Berlin Heiderberg 2001), p. 555.

[26] C.Y. Loh, L.S. Hultgren and P.C.E. Jorgenson, Near Field Screech Noise Computation for An Underexpanded Supersonic Jet by the CE/SE Method, AIAA Paper 2001-2252 (2001).

[27] X.Y. Wang, S.C. Chang, and P.C.E. Jorgenson, Numerical Simulation of Aeroacoustic Field in a 2D Cascade Involving a Downstream Moving Grid Using the Space-Time CE/SE method, in Proceedings of the First International Conference on Computational Fluid Dynamics, Kyoto, Japan, 10-14 July, 2000, edited by N. Satofuka, (Springer-Verlag Berlin Heiderberg 2001), p. 543.

[28] X.Y. Wang, S.C. Chang, A. Himansu, and P.C.E. Jorgenson, Gust Acoustic Response of A Single Airfoil Using the Space-Time CE/SE Method, AIAA Paper 2002-0801 (2002).

[29] S.T. Yu and S.C. Chang, Treatments of Stiff Source Terms in Conservation Laws by the Method of Space-Time Conservation Element and Solution Element, AIAA Paper 97-0435 (1997).

[30] S.T. Yu and S.C. Chang, Applications of the Space-Time Conservation Element / Solution Element Method to Unsteady Chemically Reactive Flows, AIAA Paper 97-2099, in A Collection of Technical Papers, 13th AIAA CFD Conference, June 29-July 2, 1997, Snowmass, CO.

[31] S.T. Yu, S.C. Chang, P.C.E. Jorgenson, S.J. Park and M.C. Lai, Treating Stiff Source Terms in Conservation Laws by the Space-Time Conservation Element and Solution Element Method, in Proceedings of the 16th International Conference on Numerical Method in Fluid Dynamics, Arcachon, France, 6-10 July, 1998, edited by C.H. Bruneau, (Springer-Verlag Berlin Heidelberg 1998), p. 433.

[32] X.Y. Wang and S.C. Chang, A 3D structured/unstructured Euler solver based on the spacetime conservation element and solution element method, in A Collection of Technical Papers, 14th AIAA CFD Conference, June 28-July 1, 1999, Norfolk, Virginia, AIAA Paper 99-3278. 
[33] N.S. Liu and K.H. Chen, Flux: An Alternative Flow Solver for the National Combustion Code, AIAA Paper 99-1079.

[34] G. Cook, High Accuracy Capture of Curved Shock Front Using the Method of Conservation Element and Solution Element, AIAA Paper 99-1008.

[35] S.C. Chang, Y. Wu, X.Y. Wang, and V. Yang, Local Mesh Refinement in the Space-Time CE/SE Method, in Proceedings of the First International Conference on Computational Fluid Dynamics, Kyoto, Japan, 10-14 July, 2000, edited by N. Satofuka, (Springer-Verlag Berlin Heiderberg 2001), p. 61.

[36] S.C. Chang, Z.C. Zhang, S.T. John Yu, and P.C.E. Jorgenson, A Unified Wall Boundary Treatment for Viscous and Inviscid Flows in the CE/SE Method, in Proceedings of the First International Conference on Computational Fluid Dynamics, Kyoto, Japan, 10-14 July, 2000, edited by N. Satofuka, (Springer-Verlag Berlin Heiderberg 2001), p. 671.

[37] Z.C. Zhang, S.T. John Yu, S.C. Chang, and P.C.E. Jorgenson, Calculations of Low-MachNumber Viscous Flows without Preconditioning by the Space-Time CE/SE method, in Proceedings of the First International Conference on Computational Fluid Dynamics, Kyoto, Japan, 10-14 July, 2000, edited by N. Satofuka, (Springer-Verlag Berlin Heiderberg 2001), p. 127.

[38] A. Himansu, P.C.E. Jorgenson, X.Y. Wang, and S.C. Chang, Parallel CE/SE Computational via Domain Decomposition, in Proceedings of the First International Conference on Computational Fluid Dynamics, Kyoto, Japan, 10-14 July, 2000, edited by N. Satofuka, (SpringerVerlag Berlin Heiderberg 2001), p. 423.

[39] Y. Wu, V. Yang, and S.C. Chang, Space-Time Method for Chemically Reacting Flows with Detailed Kinetics, in Proceedings of the First International Conference on Computational Fluid Dynamics, Kyoto, Japan, 10-14 July, 2000, edited by N. Satofuka, (Springer-Verlag Berlin Heiderberg 2001), p. 207.

[40] Other CE/SE references are posted on the web site: http://www.grc.nasa.gov/www/microbus.

[41] R. Courant and K.O. Friedrichs, Supersonic Flow and Shock Waves, Interscience, New York, 1948.

[42] G.A. Sod, A Survey of several Finite Difference Methods for Systems of Nonlinear Hyperbolic Conservation Laws, J. Comput. Phys., 27, 1 (1978).

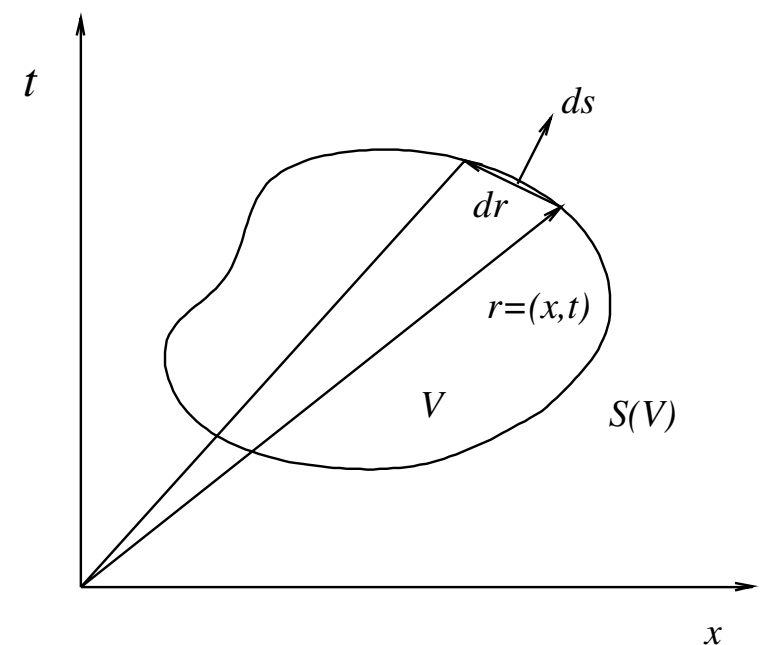

Figure 1. A surface element on the boundary $\mathrm{S}(\mathrm{V})$ of an arbitrary space-time volume $\mathrm{V}$. 


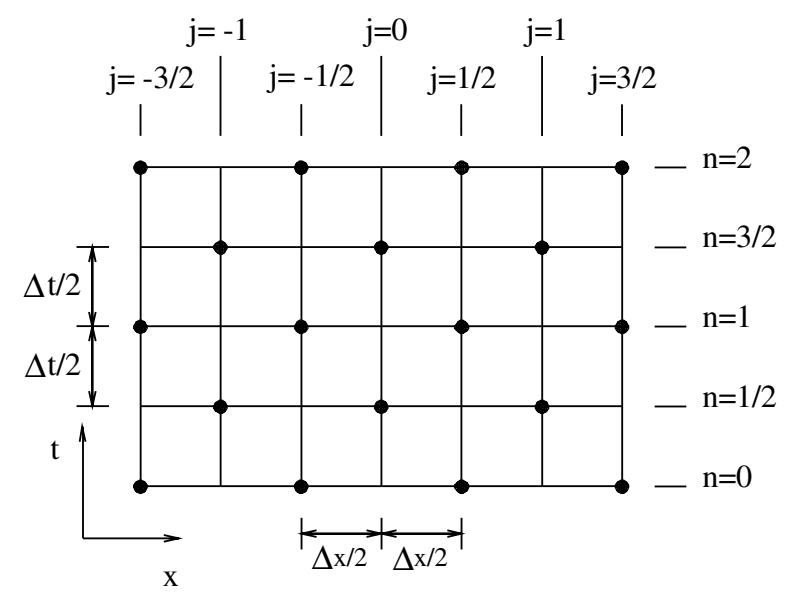

2(a). - The space-time mesh.

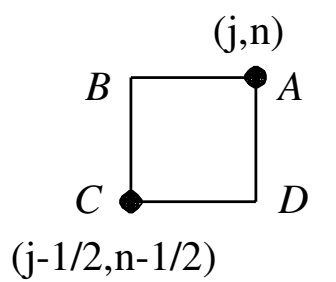

2(c). $\mathrm{CE}_{-}(\mathrm{j}, \mathrm{n})$

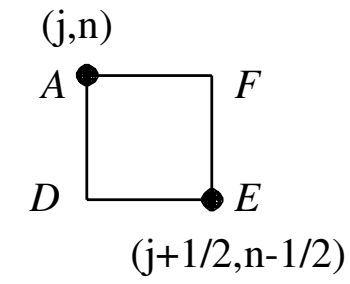

2(d). $\mathrm{CE}_{+}(\mathrm{j}, \mathrm{n})$

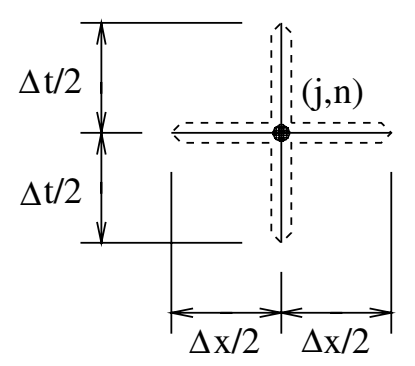

2(b). $-\operatorname{SE}(j, n)$

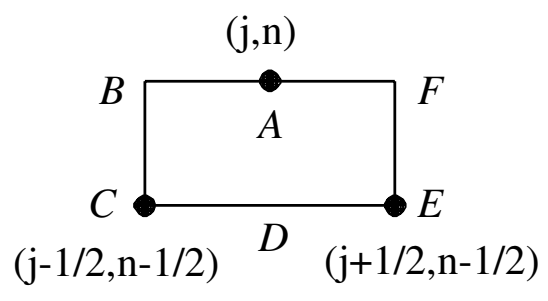

2(e). $\mathrm{CE}(\mathrm{j}, \mathrm{n})$

Figure 2. - The SEs and CEs. 


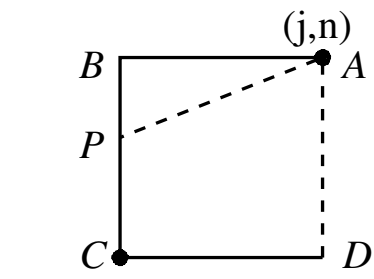

(j-1/2,n-1/2)

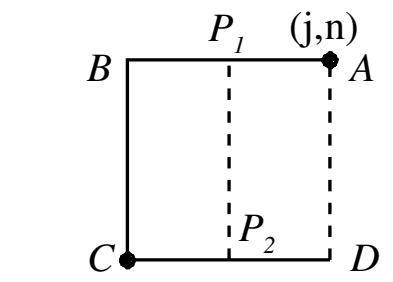

$(\mathrm{j}-1 / 2, \mathrm{n}-1 / 2)$ (b)

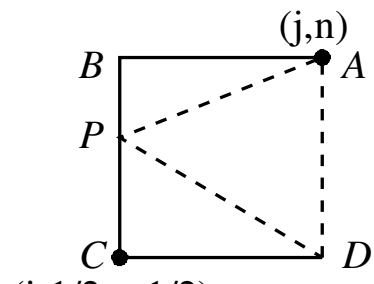

$(\mathrm{j}-1 / 2, \mathrm{n}-1 / 2)$

(c)

Figure 3. - Generalized Conservation Elements.

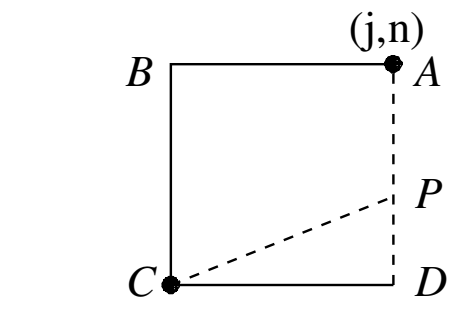

(j-1/2,n-1/2)

Figure 4. - Ill-defined Generalized

Conservation Elements.

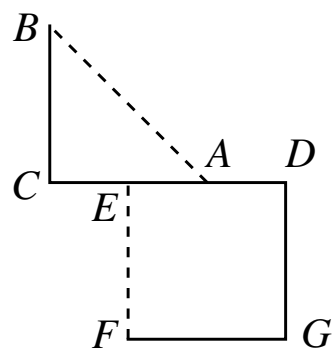

(a)

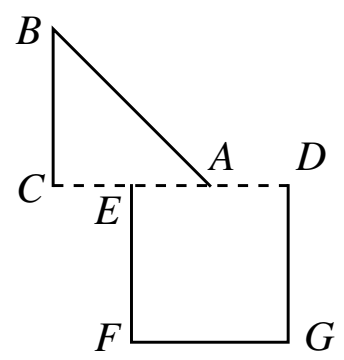

(b)

Figure 5. - Unions of Generalized Conservation Elements. 


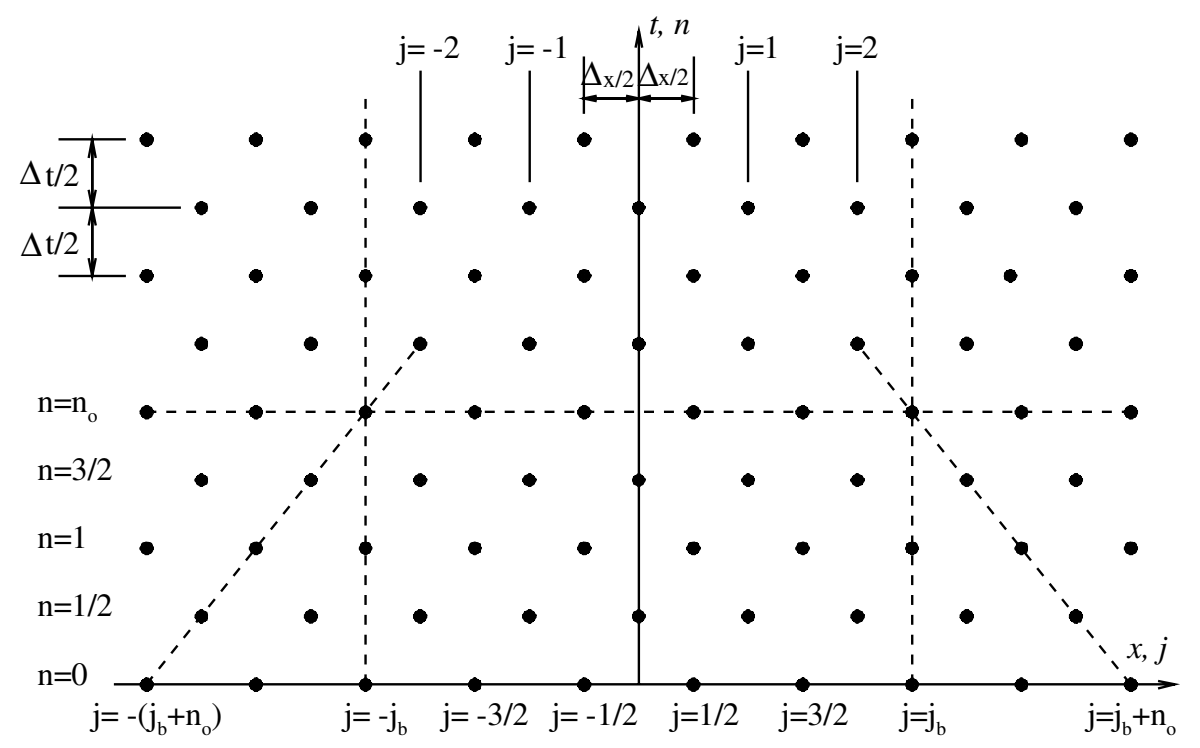

Figure 6. - An unbounded space-time mesh $\left(\mathrm{j}_{\mathrm{o}}=\mathrm{n}_{\mathrm{o}}=2\right)$.

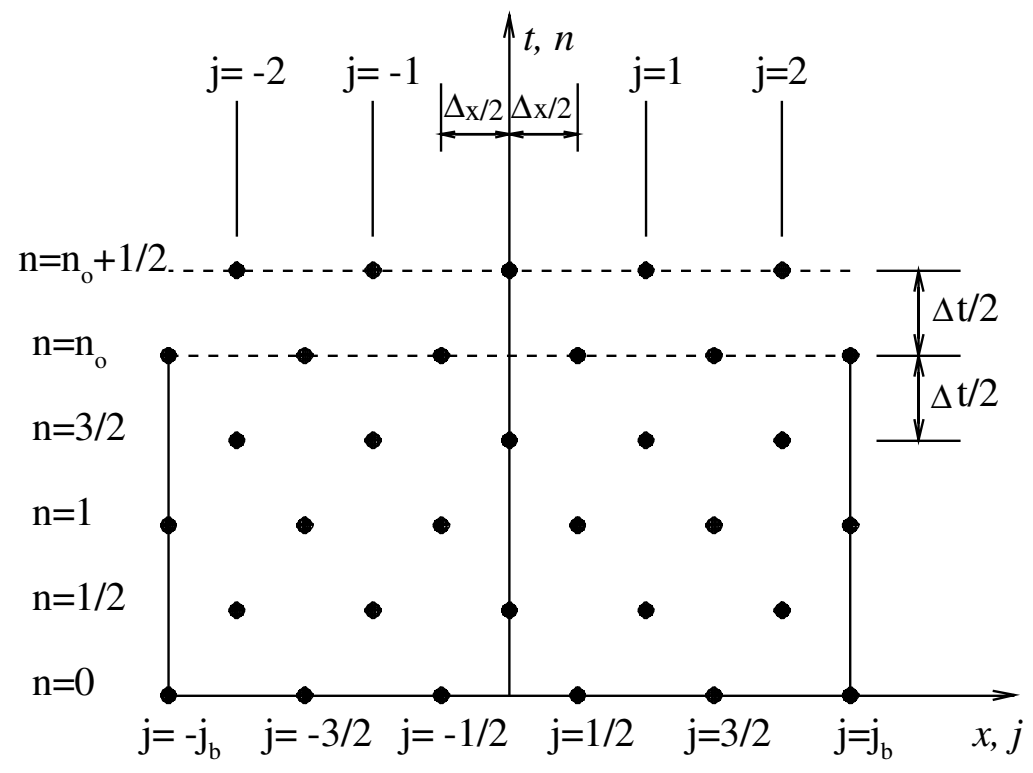

Figure 7. - A bounded space-time mesh $\left(\mathrm{j}_{\mathrm{o}}=\mathrm{n}_{\mathrm{o}}=2\right)$. 


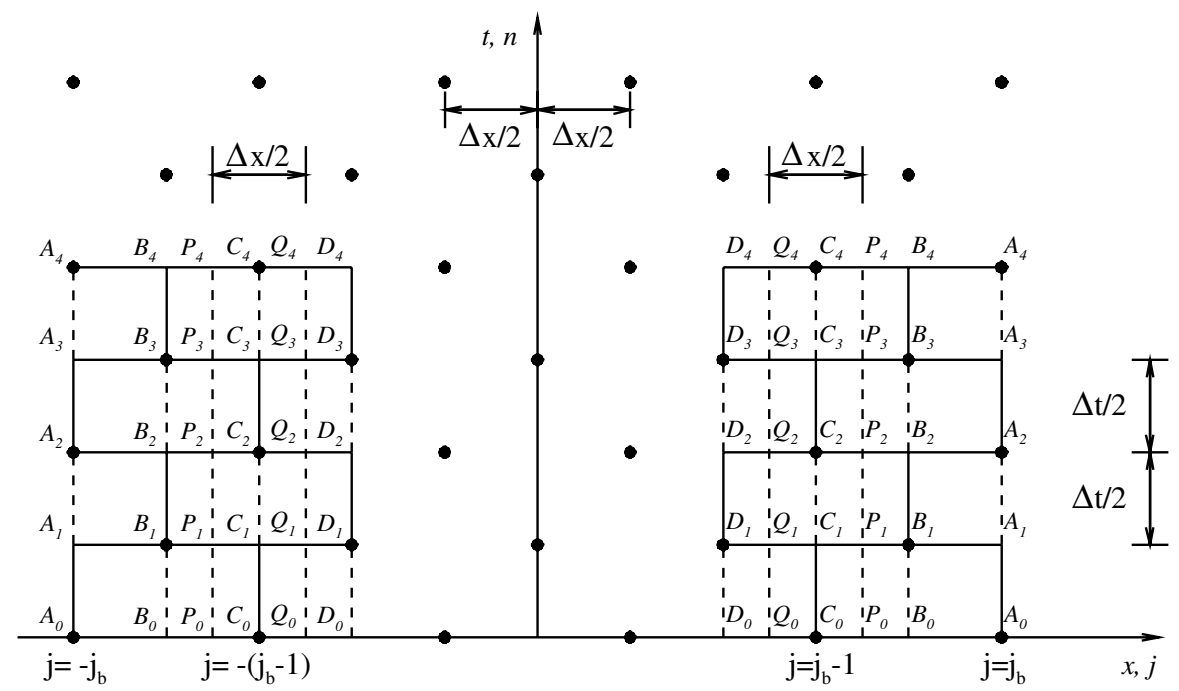

Figure 8. - GCEs used in the construction of the first, second and fourth sets of non-reflecting boundary conditions.

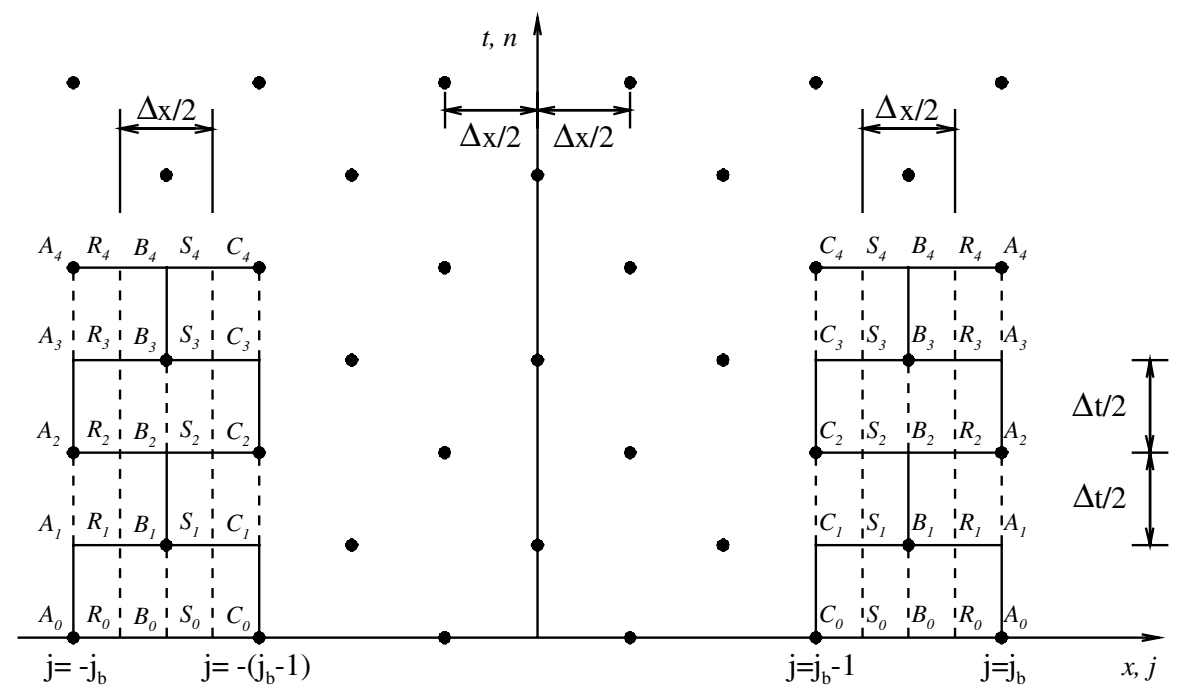

Figure 9. - GCEs used in the construction of the third set of non-reflecting boundary conditions. 

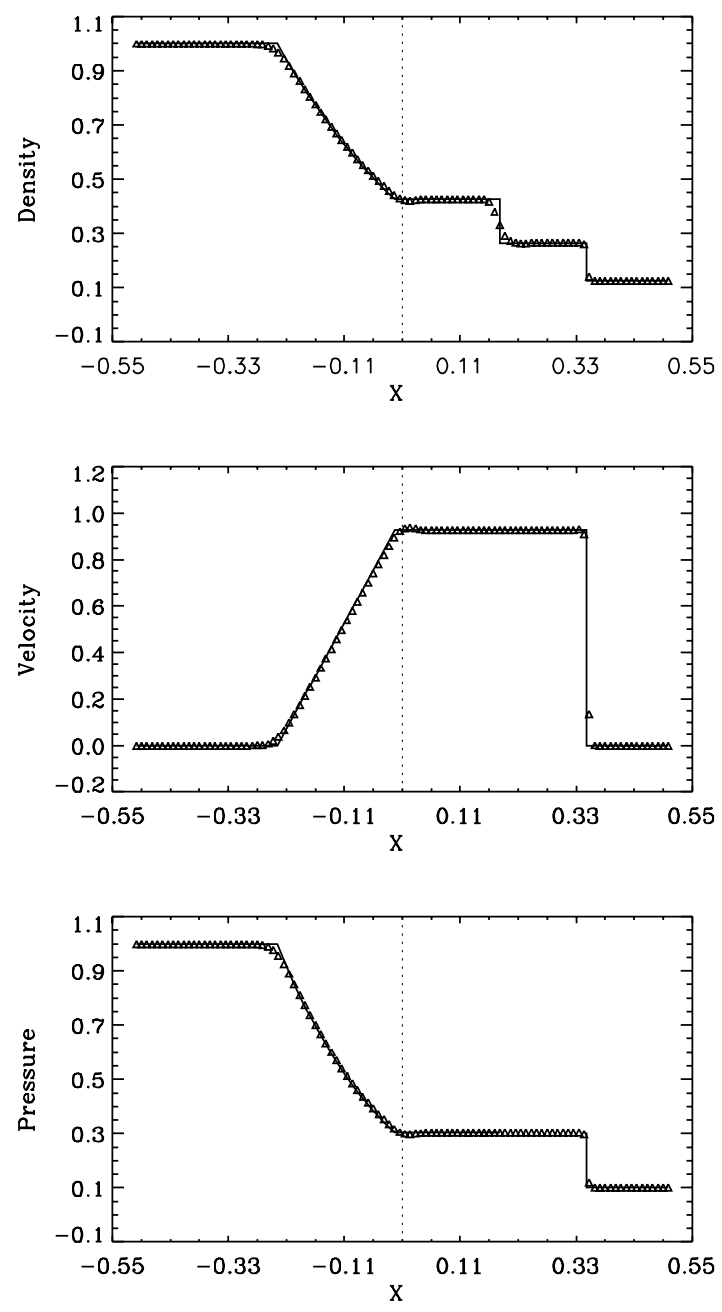

(a) Profiles at $t=0.2$

Figure 10. $=\mathrm{CE} / \mathrm{SE}$ solution using the first set of NRBCs

$$
(\Delta x=0.01, \mathrm{CFL}=0.88, \alpha=1) .
$$



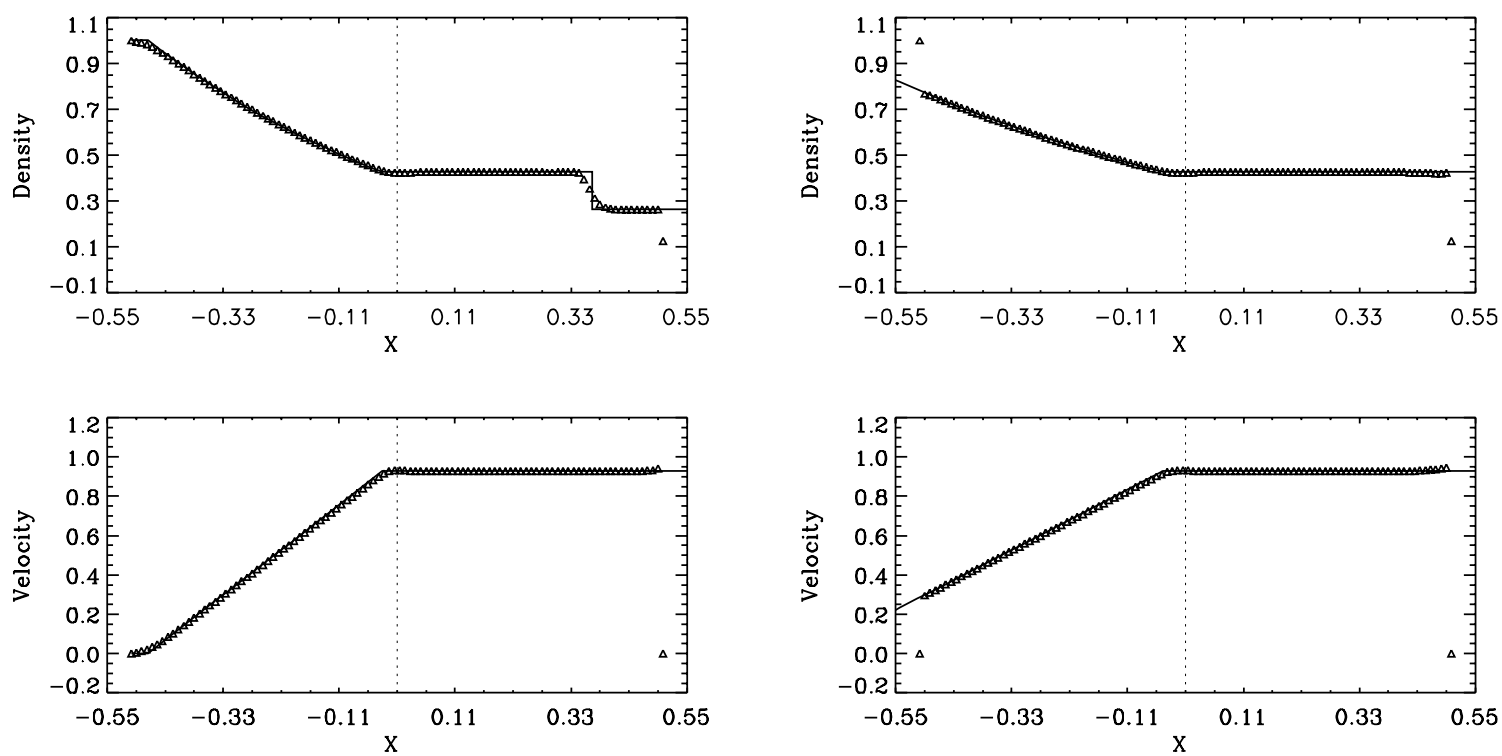

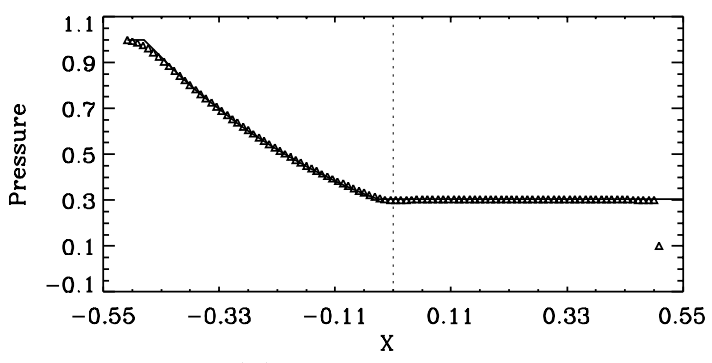

(b) Profiles at $t=0.4$

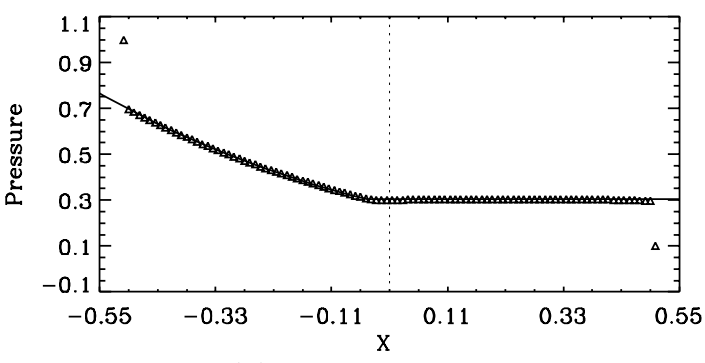

(c) Profiles at $t=0.6$

Fig. 10 (Continued) 

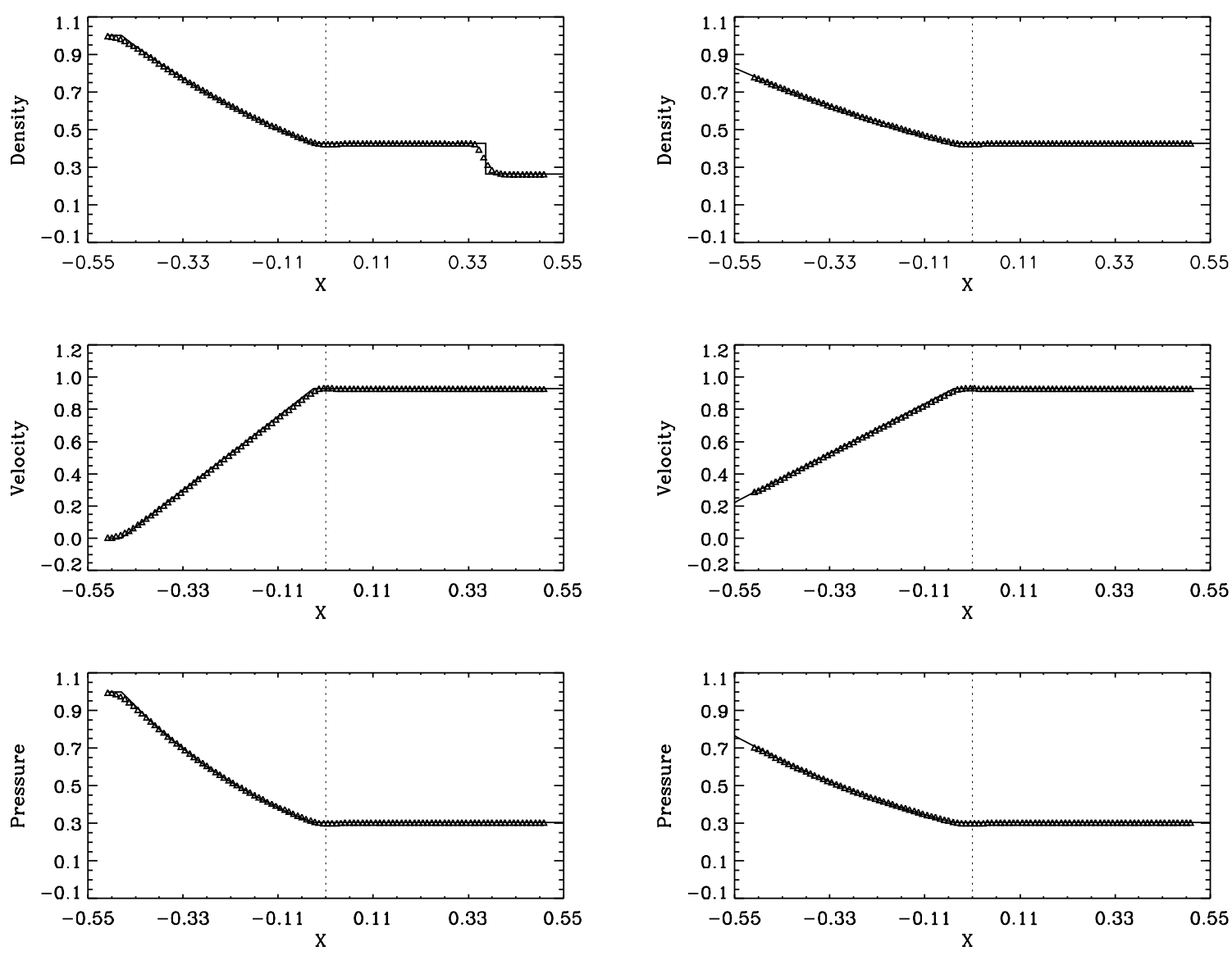

(a) Profiles at $t=0.4$

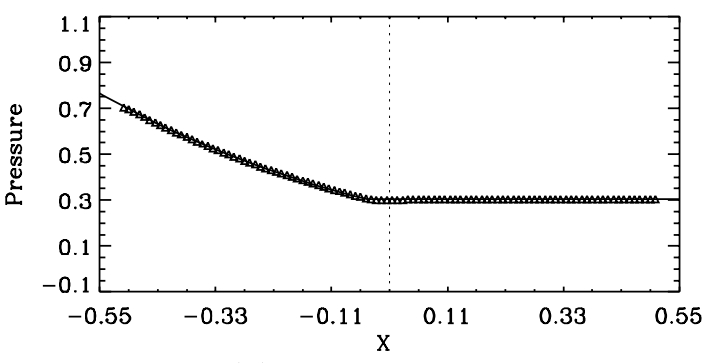

(b) Profiles at $t=0.6$

Figure 11. - CE/SE solution using the third set of NRBCs with $\lambda=\lambda^{\prime}=0(\Delta x=0.01, \mathrm{CFL}=0.88, \alpha=1)$ 

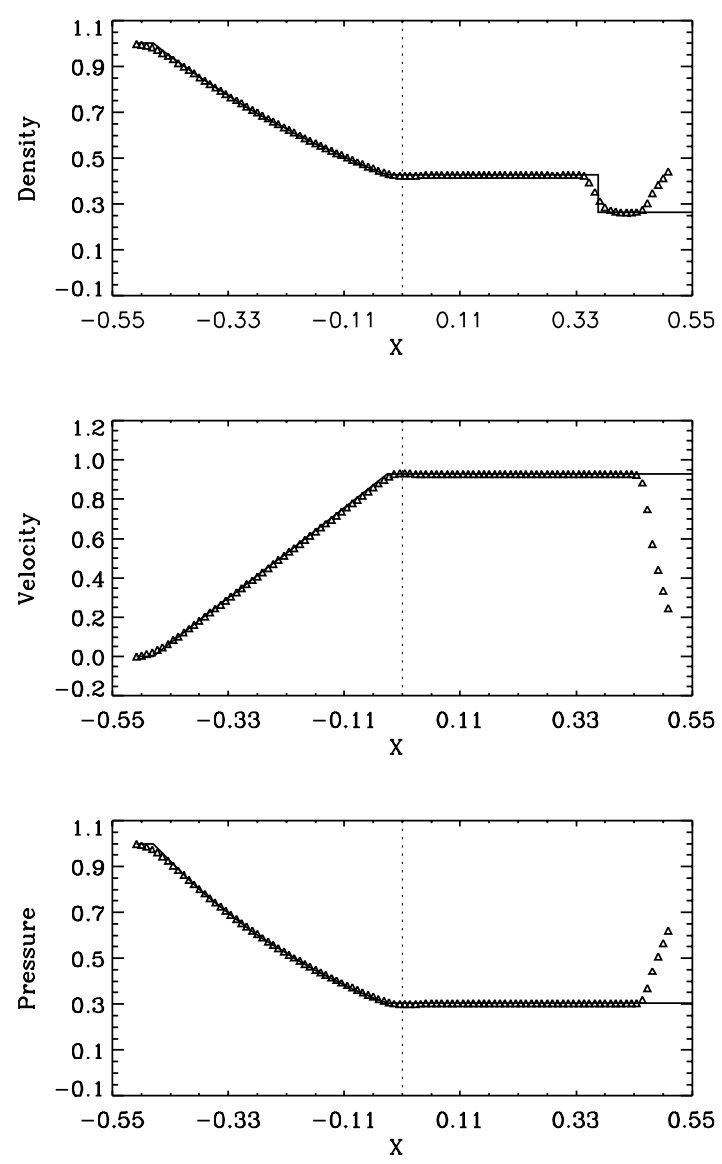

Figure 12. $-\mathrm{CE} / \mathrm{SE}$ solution at $t=0.4$ using the boundary conditions Eqs. (5.5) and (5.6) $(\Delta x=0.01, \mathrm{CFL}=0.88, \alpha=1)$ 


\section{Author Contact Information}

NASA GlenN RESEARCh CENTER, Cleveland, OH 44135

Current address: NASA Glenn Research Center, Cleveland, OH 44135

E-mail address: sin-chung.chang@grc.nasa.gov

TAITECh Inc. AND NASA GlenN Research CENTER, Cleveland, OH 44135

E-mail address: ananda.himansu@grc.nasa.gov

Taitech Inc. and NASA Glenn Research Center, Cleveland, OH 44135

E-mail address: ching.y.loh@grc.nasa.gov

Taitech Inc. and NASA Glenn Research Center, Cleveland, OH 44135

E-mail address: wangxy@spacetime.risc.rockwell.com

WAyne State UnIVERsity, Detroit, MI 48202

E-mail address: styu@me1.eng.wayne.edu 
Public reporting burden for this collection of information is estimated to average 1 hour per response, including the time for reviewing instructions, searching existing data sources, gathering and maintaining the data needed, and completing and reviewing the collection of information. Send comments regarding this burden estimate or any other aspect of this collection of information, including suggestions for reducing this burden, to Washington Headquarters Services, Directorate for Information Operations and Reports, 1215 Jefferson Davis Highway, Suite 1204, Arlington, VA 22202-4302, and to the Office of Management and Budget, Paperwork Reduction Project (0704-0188), Washington, DC 20503.

\begin{tabular}{|l|l|l|}
\hline 1. AGENCY USE ONLY (Leave blank) & $\begin{array}{c}\text { 2. REPORT DATE } \\
\text { July } 2003\end{array}$ & $\begin{array}{r}\text { 3. REPORT TYPE AND DATES COVERED } \\
\text { Technical Memorandum }\end{array}$ \\
\hline
\end{tabular}

4. TITLE AND SUBTITLE

5. FUNDING NUMBERS

Robust and Simple Non-Reflecting Boundary Conditions for the Euler

Equations-A New Approach Based on the Space-Time CE/SE Method

6. AUTHOR(S)

WBS-22-708-48-03

Sin-Chung Chang, Ananda Himansu, Ching-Yuen Loh, Xiao-Yen Wang, and Shang-Tao John Yu

7. PERFORMING ORGANIZATION NAME(S) AND ADDRESS(ES)

National Aeronautics and Space Administration

John H. Glenn Research Center at Lewis Field

Cleveland, Ohio 44135-3191

8. PERFORMING ORGANIZATION

REPORT NUMBER

E-14051

9. SPONSORING/MONITORING AGENCY NAME(S) AND ADDRESS(ES)

10. SPONSORING/MONITORING

AGENCY REPORT NUMBER

National Aeronautics and Space Administration

Washington, DC 20546-0001

NASA TM-2003-212495

\section{SUPPLEMENTARY NOTES}

Sin-Chung Chang, NASA Glenn Research Center; Ananda Himansu, Ching-Yuen Loh, Xiao-Yen Wang, Taitech, Inc., Beaver Creek, Ohio 45430; Shang-Tao John Yu, Wayne State University, Detroit, Michigan 48202. Responsible person, Sin-Chung Chang, organization code 5880, 216-433-5874.

12a. DISTRIBUTION/AVAILABILITY STATEMENT

12b. DISTRIBUTION CODE

Unclassified - Unlimited

Subject Categories: 34 and 64

Distribution: Nonstandard

Available electronically at http://gltrs.grc.nasa.gov

This publication is available from the NASA Center for AeroSpace Information, 301-621-0390.

13. ABSTRACT (Maximum 200 words)

This paper reports on a significant advance in the area of non-reflecting boundary conditions (NRBCs) for unsteady flow computations. As a part of the development of the space-time conservation element and solution element (CE/SE) method, sets of NRBCs for 1D Euler problems are developed without using any characteristics-based techniques. These conditions are much simpler than those commonly reported in the literature, yet so robust that they are applicable to subsonic, transonic and supersonic flows even in the presence of discontinuities. In addition, the straightforward multidimensional extensions of the present 1D NRBCs have been shown numerically to be equally simple and robust. The paper details the theoretical underpinning of these NRBCs, and explains their unique robustness and accuracy in terms of the conservation of space-time fluxes. Some numerical results for an extended Sod's shock-tube problem, illustrating the effectiveness of the present NRBCs are included, together with an associated simple Fortran computer program. As a preliminary to the present development, a review of the basic CE/SE schemes is also included.

14. SUBJECT TERMS

Euler equations of motion

17. SECURITY CLASSIFICATION OF REPORT

Unclassified
18. SECURITY CLASSIFICATION OF THIS PAGE

Unclassified
19. SECURITY CLASSIFICATION OF ABSTRACT

Unclassified
15. NUMBER OF PAGES

39

16. PRICE CODE

20. LIMITATION OF ABSTRACT

Standard Form 298 (Rev. 2-89)

Prescribed by ANSI Std. Z39-18 298-102 